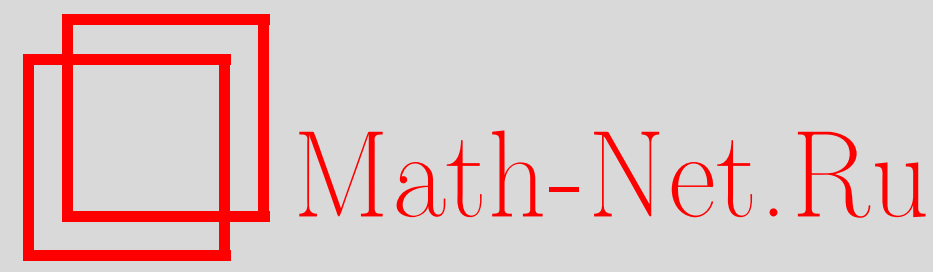

И. В. Микитюк, Кэлеровы структуры на касательных расслоениях симметрических пространств ранга один, Матем. сб., 2001, том 192, номер 11, 93-122

DOI: https://doi.org/10.4213/sm611

Использование Общероссийского математического портала Math-Net.Ru подразумевает, что вы прочитали и согласны с пользовательским соглашением http://www . mathnet.ru/rus/agreement

Параметры загрузки:

IP: 54.81 .137 .203

26 апреля 2023 г., 16:50:29 
УДК 514.765.1+512.813.4

\author{
И.В. Микитюк
}

\title{
Кэлеровы структуры на касательных расслоениях симметрических пространств ранга один
}

\begin{abstract}
В работе построены все $G$-инвариантные кэлеровы структуры $F$ на областях симплектических многообразий $T(G / K)$, где $G / K$ - риманово симметрическое пространство ранга один размерности $\geqslant 3$ с полупростой группой Ли $G$. Показано, что этот класс $\{F\}$ кэлеровых структур инвариантен относителњно процедуры редукции. Представлен Ли-алгебраический метод описания $G$-инвариантных кэлеровых структур на касательных расслоениях симметрических пространств $G / K$. Также обсуждаются смежные вопросы описания тройной системы Ли пространства $F_{4} / \operatorname{Spin}(9)$ в терминах его спинорной структуры.

Библиографйл: 18 названий.
\end{abstract}

\section{§1. Введение}

Пусть $G / K$ - симметрическое пространство с полупростой группой Ли $G$ и компактной подгруппой $K$. Стандартная $G$-инвариантная метрика $g$ на $G / K$ определяет геодезический поток с гамильтонианом $H$ на касательном расслоении $T(G / K)$, рассматриваемом как симплектическое многообразие с симплектической 2-формой $\Omega$ (индуцированной канонической симплектической структурой на кокасательном расслоении после отождествления этих двух расслоений с помощью метрики).

Комплексные структуры, определенные на выколотом касательном расслоении $T^{0}(G / K)=T(G / K)-\{$ нулевое сечение $\}$, естественно возникают как результат геометрических конструкций метода геометрического квантования. Такую структуру для сферы обнаружил Сурио в работе [1]. Позже Раунсли [2] заметил, что функция длины $\sqrt{H}$ является строго плюрисубгармонической относительно упомянутой выше комплексной структуры $J_{S}$ и, таким образом, определяет кэлерову метрику на $T^{0} S^{n}$ с $\Omega$ как кэлеровой формой. Он также заметил, что $J_{S}$ инвариантна относительно гамильтонова потока $X \sqrt{H}$ функции длины $\sqrt{H}$ (нормализованного геодезического потока) и использовал кэлерову структуру $J_{S}$ для квантования.

Впоследствии Фурутани и Танака [3] определили кэлерову структуру $J_{S}$ с аналогичными свойствами на выколотых касательных расслоениях комплексного и кватернионного проективных пространств $\mathbb{C} P^{n}, \mathbb{H} P^{n}$ и использовали ее для квантования. В работе [4] Ии и Морикава описали эту структуру (на выколотом касательном расслоении) в терминах геометрических структур, ассоциированных с метрикой $g$ на $G / K$.

Работа выполнена при финансовой поддержке Национального научного фонда Германии (грант № SFB 237).

(C) и.В. Микитюк 2001 
В работе [5] Шоке исследовал связь между $J_{S}$ и так называемой адаптированной комплексной структурой $J_{A}$ на соответствующем касательном расслоении $T(G / K)$. Он показал, что для всех компактных симметрических пространств ранга один (включая проективную плоскость Кэли $\left.F_{4} / \operatorname{Spin}(9)\right)$ семейство комплексных структур, являющихся образами адаптированной комплексной структуры относительно подходящего семейства диффеоморфизмов, имеет гранищу и эта граничная комплексная структура совпадает с $J_{S}$. В нашей работе [6] мы, используя методы теории алгебр Ли, описали все $G$-инвариантные кэлеровы структуры $F$ (с $\Omega$ как кэлеровой формой) на вьколотых касательных расслоениях $T^{0}(G / K)$ симметрических пространств $G / K$, которые инвариантны относительно нормализованного геодезического потока $X_{\sqrt{H}}$. Мы показали, что такие кэлеровы структуры $F$ существуют только на выколотых касательных расслоениях компактных симметрических пространств ранга один.

В настоящей работе представлен метод описания $G$-инвариантных кэлеровых структур на касательных расслоениях симметрических пространств $G / K$, основанный на теории алгебр Ли. Этот метод базируется на подходе работы [6] и на обобшении упомянутого вьше метода, изложенного в [5]. Главная цель нашей работы состоит в построении всех $G$-инвариантных кэлеровых структур $F$ на $G$-инвариантных областях касательных расслоений $T(G / K)$ симметрических пространств $G / K$ ранга один размерности $\geqslant 3$ (теорема 4.14$)$. Доказано, что такие кэлеровы структуры $F$ определяются парой комплекснозначных гладких функций $(\psi, \lambda)$, связанных уравнением Риккати (4.4), и что этот класс $\{F\}$ кэлеровых структур инвариантен относительно процедуры редукции. Как приложение методов, развитых в $\S 3$, получено описание тройной системы Ли симметрического пространства $F_{4} / \operatorname{Spin}(9)$ в терминах его спинорной структуры.

Часть этой работы была выполнена автором во время его пребывания в Рурском университете (Бохум, Германия) в апреле-мае 2000 года. Автор выражает благодарность А. Т. Хаклберри за поддержку, гостеприимство и плодотворные дискуссии.

\section{§2. $G$-инвариантные кэлеровы структуры на $T(G / K)$}

2.1. Поляризации. Здесь мы приведем необходимые в дальнейшем факты о поляризациях. Более детальное изложение этого материала можно найти, например, в работах [7], [8].

Пусть $(X, \Omega)$ - вешественное симплектическое многообразие, $F$ - комплексное распределение, т.е. комплексноеподрасслоение комплексифицированного касательного расслоения $T^{\mathbb{C}} X$. Распределение $F$ определяет комплексную структу$p y$ на $X$, если $F$ инволютивно (т.е. замкнуто относительно скобки Ли: $[F, F] \subset F)$, $F \cap \bar{F}=0$ и $F+\bar{F}=T^{\mathbb{C}} X$. Говорят, что распределение $F$ интегрируемо, если

(1) $F \cap \bar{F}$ имеет постоянный ранг,

(2) распределения $F$ и $F+\bar{F}$ инволютивны.

Распределение $F$ лагранжсево, если $\Omega(F, F)=0$ и $\operatorname{dim}_{\mathbb{C}} F=\frac{1}{2} \operatorname{dim}_{\mathbb{R}} X$.

Поляризаиия на $X$ - это комплексное интегрируемое лагранжево распределение $F \subset T^{\mathbb{C}} X$. Поляризация $F$ положсительно определена, если $-i \Omega_{x}(Z, \bar{Z})>0$ для всех ненулевых векторов $Z \in F(x)$ и $x \in X$. 
ОПРЕДЕЛЕНИЕ 2.1 [8]. Кәлерово многообразие - это симплектическое многообразие, снабженное положительно определенной поляризацией.

Чтобы согласовать это определение с общепринятьм, мы будем использовать следующую лемму (см. [8; лемма 4.3]).

ЛЕмма 2.2. Пусть $F$ - полохсительно определенная поляризачия на вещественном симплектическом многообразии $X$. Тогда для каждого $x \in X$ существует единственное линейное отображение $J_{x}: T_{x} X \rightarrow T_{x} X$ такое, чmo

(1) $J_{x}^{2}=-\operatorname{Id}_{x}$;

(2) $F(x)=\left\{Y+i J_{x}(Y), Y \in T_{x} X\right\}$;

(3) $\Omega_{x}\left(J_{x}\left(Y_{1}\right), J_{x}\left(Y_{2}\right)\right)=\Omega_{x}\left(Y_{1}, Y_{2}\right)$ для произвольных $Y_{1}, Y_{2} \in T_{x} X$;

(4) квадратичная форма $B_{x}\left(Y_{1}, Y_{2}\right)=\Omega_{x}\left(J_{x} Y_{1}, Y_{2}\right)$ симметрична и положительно определена.

Из леммы 2.2 следует, что $F$ - (интегрируемое) комплексное распределение, состоящее из векторов типа $(0,1)$ комплексной структуры $J$, а четверка $(X, J, B, \Omega)$ является кэлеровым многообразием в обычном понимании этого термина.

2.2. $G$-инвариантные комплексные структуры. В этом пункте мы изложим некоторые результаты работы [6]. Пусть $M$ - однородное пространство вещественной связной редуктивной группы Ли $G$, т.е. $M=G / K$. Предположим, что $K$ - (замкнутая) редуктивная подгруппа групшы $G$. Обозначим через $\mathfrak{g}$ и k алгебры Ли групп Ли $G$ и $K$ соответственно. Тогда существует точное представление алгебры Ли $\mathfrak{g}$ такое, что ассоциированная с ним билинейная форма $\Phi$ невырождена на $\mathfrak{g}$ (если $\mathfrak{g}$ полупроста, то в качестве $\Phi$ мы можем взять форму Киллинга, ассоциированную с присоединенньм представлением алгебры Ли $\mathfrak{g})$. Форма $\Phi$ невырождена на k. Пусть $\langle\cdot, \cdot\rangle=c \Phi$, где $c \in \mathbb{R}$ - ненулевая константа. Форма $\langle\cdot, \cdot\rangle$ определяет $G$-инвариантную псевдориманову метрику $g$ на многообразии $G / K$. Метрика $g$ позволяет отождествить кокасательное расслоение $T^{*} M$ и касательное расслоение $T M$, и, таким образом, мы можем говорить о канонической 1-форме $\theta$ на $T M$, т.е. форме, определенной уравнением

$$
\theta(Y) \stackrel{\text { def }}{=} g\left(x, p_{*} Y\right), \quad Y \in T_{x}(T M)
$$

где $p: T M \rightarrow M$ обозначает естественную проекцию. Форма $\theta$ и симплектическая форма $\Omega \stackrel{\text { def }}{=} d \theta$ инвариантны относительно естественного действия групшы Ли $G$ на $T M$ (продолжения действия $G$ на $M$ ).

Обозначим через $\mathfrak{m}$ ортогональное дополнение к $\mathfrak{k}$ в $\mathfrak{g}$ относительно $\langle\cdot, \cdot\rangle$, т.е. $\mathfrak{g}=\mathfrak{k} \oplus \mathfrak{m}-$ Ad $K$-инвариантное разложение $\mathfrak{g}$ в прямую сумму подпространств. Рассмотрим тривиальное векторное расслоение $G \times \mathfrak{m}$ и на нем два коммутирующих действия групп Ли: действие группы Ли $G$ слева $l_{h}:(g, w) \mapsto(h g, w)$ и действие группы Ли $K$ справа $r_{k}:(g, w) \mapsto\left(g k, \operatorname{Ad} k^{-1}(w)\right)$. Пусть $\pi: G \times \mathfrak{m} \rightarrow$ $G \times_{K} \mathfrak{m}$ - естественная проекция. Хорошо известно, что пространства $G \times_{K} \mathfrak{m}$ и $T M$ изоморфны. Используя соответствуюший $G$-эквивариантньй диффеоморфизм $\varphi: G \times_{K} \mathfrak{m} \rightarrow T M,\left.[(g, w)] \mapsto \frac{d}{d t}\right|_{0} g \exp (t w) K$, и проекцию $\pi$, определим 
$G$-эквивариантную субмерсию $\Pi: G \times \mathfrak{m} \rightarrow T M, \Pi=\varphi \circ \pi$. Обозначим через $\widetilde{\theta}$ 1-форму $\Pi^{*} \theta$, а через $\widetilde{\Omega}$ - ее дифференциал $d \widetilde{\theta}$. Пусть $\xi^{l}-$ левоинвариантное векторное поле на $G$, определяемое вектором $\xi \in \mathfrak{g}$.

ЛЕмма 2.3. 1-форма $\widetilde{\theta}$ на многообразии $G \times \mathfrak{m}$ имеет вид

$$
\begin{aligned}
\widetilde{\theta}_{(g, w)}\left(\xi^{l}(g), u\right) & =\langle w, \xi\rangle \\
\widetilde{\Omega}_{(g, w)}\left(\left(\xi_{1}^{l}(g), u_{1}\right),\left(\xi_{2}^{l}(g), u_{2}\right)\right) & =\left\langle\xi_{2}, u_{1}\right\rangle-\left\langle\xi_{1}, u_{2}\right\rangle-\left\langle w,\left[\xi_{1}, \xi_{2}\right]\right\rangle,
\end{aligned}
$$

əде $g \in G, w \in \mathfrak{m}, \xi, \xi_{1}, \xi_{2} \in \mathfrak{g}, u, u_{1}, u_{2} \in \mathfrak{m}=T_{w} \mathfrak{m} . \quad$ Ядро $\mathscr{K} \subset T(G \times \mathfrak{m})$ 2-формы $\widetilde{\Omega}$ порождается глобальными (лево) $G$-инвариантными векторными полями $\zeta^{L}, \zeta \in \mathfrak{k}$, на $G \times \mathfrak{m}, \zeta^{L}(g, w)=\left(\zeta^{l}(g),[w, \zeta]\right)$.

ДокАЗАТЕльство. Выражение для 1-формы $\widetilde{\theta}$ следует непосредственно из определения (2.1). Чтобы получить формулу для $\widetilde{\Omega}$, зафиксируем базис $\left\{\eta_{j}\right\}$ в $\mathfrak{g}$ и соответствуюший дуальный базис $\left\{\omega_{j}\right\}$ в $\mathfrak{g}^{*}$. Тогда $\widetilde{\theta}=\sum_{j} f_{j} \omega_{j}^{L}$, где $f_{j}(g, w)=$ $\left\langle w, \eta_{j}\right\rangle$ - гладкая функция и $\omega_{j}^{L}-$ поднятие вдоль проекции $G \times \mathfrak{m} \rightarrow G$ (лево) $G$-инвариантной 1-формы $\omega_{j}^{l}$ на группе Ли $G$. Теперь достаточно заметить, что $d f_{j}(g, w)\left(\xi^{l}(g), u\right)=\left\langle u, \eta_{j}\right\rangle$, и применить формулу Маурера-Картана: $d \omega_{j}^{l}\left(\xi^{l}, \eta^{l}\right)=$ $-\omega_{j}([\xi, \eta])$. Так как $\Omega$ является симплектической формой, ядро формы $\widetilde{\Omega}$ совпадает с ядром отображения $\Pi_{*}$.

Пусть $D$ - открытое связное $G$-инвариантное подмножество в $T M$. Обозначим через $W$ единственное $\mathrm{Ad} K$-инвариантное открытое подмножество в $\mathfrak{m}$ такое, что $\Pi^{-1}(D)=G \times W$, а через $\mathscr{K}_{W}$ - ограничение распределения $\mathscr{K}$ на открытое подмногообразие $G \times W$.

Пусть $F$ - поляризация на $D$. Так как $F$ - интегрируемое распределение, то оно определяется дифференциальным идеалом $\mathscr{I}(F) \subset \Lambda T^{\mathbb{C} *} D$ (замкнутым относительно операции внешнего дифференцирования). Ядро $\mathscr{F}$ дифференциального идеала $\Pi^{*} \mathscr{I}(F)$ является интегрируемым комплексным распределением на $G \times W$. Распределение $\mathscr{F}$ мы будем обозначать также через $\Pi_{*}^{-1}(F)$. Это распределение единственным образом определяется двумя условиями:

(1) $\operatorname{dim}_{\mathbb{C}} \mathscr{F}=\operatorname{dim}_{\mathbb{R}} G$

(2) $\Pi_{*}(\mathscr{F})=F$.

Очевидно, что $\widetilde{\Omega}(\mathscr{F}, \mathscr{F})=0$ и $\mathscr{F}$ содержит $\mathscr{K}_{W}$. Больше того, распределение $\mathscr{F}$ (право) $K$-инвариантно.

Мы сушественно упростим все выкладки, рассматривая тривиальное векторное расслоение $G \times W$ с распределением $\mathscr{F}$ вместо $G$-инвариантного подмножества $D$ касательного расслоения $T(G / K)$ с поляризацией $F$. Для этого нам необходима

ЛЕмма 2.4. Пусть $\mathscr{F}$ - интегрируемое комплексное распределение на $G \times W$ mакое, что

(1) $\mathscr{F}$ (право) $K$-инвариантно,

(2) $\mathscr{K}_{W}^{\mathbb{C}} \subset \mathscr{F}$

(3) $\operatorname{dim}_{\mathbb{C}} \mathscr{F}=\operatorname{dim}_{\mathbb{R}} G$,

(4) $\widetilde{\Omega}(\mathscr{F}, \mathscr{F})=0$. 
Тогда $F=\Pi_{*}(\mathscr{F})$ является поляризачией на $D$.

Обратно, произвольная поляризация $F$ на $D$ определяет единственное интегрируемое распределение $\mathscr{F}=\Pi_{*}^{-1}(F)$ со свойствами (1)-(4).

ДокАЗАтЕльство. Так как распределение $\mathscr{F}$ (право) $K$-инвариантно и содержит ядро $\mathscr{K}_{W}$ отображения $\Pi_{*} \mid(G \times W)$, то его образ $F=\Pi_{*}(\mathscr{F})$-корректно определенное (комплексное) распределение на $D$ размерности $\operatorname{dim} G-\operatorname{dim} K ; F \cap \bar{F}$ имеет постоянньй ранг потому, что распределение $\mathscr{K}_{W} \subset \mathscr{F}$ вешественно. Лагранжевость распределения $F$ является непосредственным следствием условия (4). Чтобы доказать его гладкость и инволютивность, заметим, что П - субмерсия, т.е. для произвольной точки $(g, w) \in G \times W$ существуют ее окрестность $U$, координаты $x_{1}, \ldots, x_{N}$ на $U$ и координаты $x_{1}, \ldots, x_{N^{\prime}}$ на открытом множестве $U^{\prime}=\Pi(U)$ такие, что $x_{j}(g, w)=0, j=1, \ldots, N$, и отображение П $\mid U$ в этих координатах имеет вид П: $\left(x_{1}, x_{2}, \ldots, x_{N}\right) \mapsto\left(x_{1}, x_{2}, \ldots, x_{N^{\prime}}\right)$. Пусть

$$
Y\left(x_{1}, \ldots, x_{N}\right)=\sum_{j=1}^{N} a_{j}\left(x_{1}, \ldots, x_{N}\right) \frac{\partial}{\partial x_{j}}
$$

- произвольное сечение распределения $\mathscr{F} \mid U$. Распределение $\mathscr{K} \mid U$ порождено $\partial / \partial x_{j}, j=N^{\prime}+1, \ldots, N$, и $\mathscr{F} \mid U$ сохраняется этими векторньми полями $\partial / \partial x_{j}$. Поэтому гладкое векторное поле

$$
Y_{0}\left(x_{1}, \ldots, x_{N}\right)=\sum_{j=1}^{N} a_{j}\left(x_{1}, \ldots, x_{N^{\prime}}, 0, \ldots, 0\right) \frac{\partial}{\partial x_{j}}
$$

также является сечением распределения $\mathscr{F} \mid U$. Таким образом,

$$
\Pi_{*} Y_{0}\left(x_{1}, \ldots, x_{N^{\prime}}\right)=\sum_{j=1}^{N^{\prime}} a_{j}\left(x_{1}, \ldots, x_{N^{\prime}}, 0, \ldots, 0\right) \frac{\partial}{\partial x_{j}}
$$

- гладкое сечение $F \mid U^{\prime}$. (Распределение $\mathscr{F}$ сохраняется векторньп полем $\partial / \partial x_{j}$ тогда и только тогда, когда $\mathscr{F}$ сохраняется соответствующей локальной однопараметрической подгруппой [7].) Теперь инволютивность $F$ следует непосредственно из равенства (2.4). Аналогично доказьвается инволютивность распределения $\Pi_{*}(\mathscr{F}+\overline{\mathscr{F}})$, т.е. $F-$ поляризация.

ПрЕДЛОЖЕНИЕ 2.5. Пусть $F-$ поляризация на $D, \mathscr{F}=\Pi_{*}^{-1}(F)$. Тогда

(1) F G-инвариантно тогда и только тогда, когда $\mathscr{F}$ (лево) G-инвариантно;

(2) $F$ - комплексная структура на $D$ тогда и только тогда, когда $\mathscr{F} \cap \overline{\mathscr{F}}=$ $\mathscr{K}_{W}^{\mathbb{C}}\left(\mathscr{F}+\overline{\mathscr{F}}=T^{\mathbb{C}}(G \times W)\right) ;$

(3) $F$ - положстельно определенная поляризация на $D$ тогда и только тогда, когда -i $\widetilde{\Omega}(Z, \bar{Z}) \geqslant 0$ для всех векторных полей (сечений) $Z \in \Gamma \mathscr{F} u \widetilde{\Omega}(Z, \bar{Z})=0 \Leftrightarrow Z \in \Gamma \mathscr{K}_{W}^{\mathbb{C}} \subset \Gamma \mathscr{F}$. 
ДокАЗАТЕльство. Так как субмерсия П $G$-эквивариантна, то распределения $\mathscr{F}$ и $\Pi_{*}(\mathscr{F}) G$-левоинвариантны одновременно. Если $\mathscr{F} \cap \overline{\mathscr{F}}=\mathscr{K}_{W}^{\mathbb{C}}$, то $\Pi_{*}(\mathscr{F}) \cap$ $\Pi_{*}(\overline{\mathscr{F}})=0$ и, следовательно, $F$ - комплексная структура. Последнее утверждение предложения очевидно.

Таким образом, сушествует взаимно однозначное соответствие между множеством всех $G$-инвариантных поляризаций $F$ на $D$ и множеством всех интегрируемых (лево) $G$-инвариантных распределений $\mathscr{F} \subset T^{\mathbb{C}}(G \times W)$, для которых выполнены условия (1)-(4) леммы 2.4 .

Пусть $F-G$-инвариантная поляризация, $\mathscr{F}=\Pi_{*}^{-1}(F)$. Теперь мы сосредоточим наше внимание на $G$-инвариантном подраспределении распределения $\mathscr{F}$, которое будет играть важную роль в нашем дальнейшем изложении. Определим это распределение $\mathscr{P} \subset \mathscr{F}$ как дополнительное к $\mathscr{K}_{W}^{\mathbb{C}}$ (в $\left.\mathscr{F}\right) G$-инвариантное распределение, для которого $\mathscr{P}(e, w) \subset \mathfrak{m}^{\mathbb{C}} \times \mathfrak{m}^{\mathbb{C}} \subset T_{e}^{\mathbb{C}} G \times T_{w}^{\mathbb{C}} W$. Так как распределение $\mathscr{K}_{W}$ порождено векторными полями $\zeta^{L} \mid(G \times W), \zeta \in \mathfrak{k}, \zeta^{L}(g, w)=\left(\zeta^{l}(g),[w, \zeta]\right)$, то такое распределение $\mathscr{P}$ единственно. По определению $\mathscr{F}=\mathscr{K}_{W}^{\mathbb{C}}+\mathscr{P}$. Больше того, распределения $\mathscr{K}_{W}, \mathscr{P}$ (и $\mathscr{F}$ по определению) являются (право) $K$-инвариантными потому, что разложение $\mathfrak{g}=\mathfrak{k} \oplus \mathfrak{m} \operatorname{Ad} K$-инвариантно.

\section{§ 3. $G$-инвариантные кэлеровы структуры на касательных расслоениях симметрических пространств}

В этом параграфе мы продолжим изложение, используя наши предыдушие обозначения, но предположим дополнительно, что $G / K$ - симметрическое пространство $([\mathfrak{m}, \mathfrak{m}] \subset \mathfrak{k})$ и что ограничение формы $\langle\cdot, \cdot\rangle$ на $\mathfrak{m}$ положительно определено. Положим $r(w)=|w|=\sqrt{\langle w, w\rangle}$ на $\mathfrak{m}$. Пусть $\mathfrak{m}^{0}=\mathfrak{m}-\{0\}$.

3.1. $G$-инвариантные комплексные структуры на касательных расслоениях симметрических пространств. Следующие лемма и предложение описывают все положительно определенные $G$-инвариантные поляризации на $G$-инвариантных областях $D$ касательного расслоения $T M$ симметрического пространства $M$ в терминах гладкого семейства $\mathbb{C}$-линейных отображений на $\mathfrak{m}^{\mathbb{C}}$.

Лемма 3.1 [6]. Пусть $\mathscr{F}=\Pi_{*}^{-1}(F)$, әде $F-G$-инвариантная положительно определенная поляризация на $D, \mathscr{F}=\mathscr{K}_{W}^{\mathbb{C}}+\mathscr{P}$. Предположим, что $G / K$ - симметрическое пространство $([\mathfrak{m}, \mathfrak{m}] \subset \mathfrak{k})$. Тогда для каждого $w \in W$ существует единственное невырохсденное $\mathbb{C}$-линейное ото-

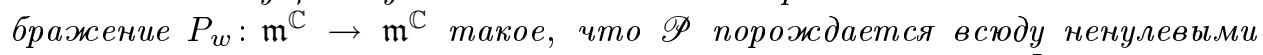
на $G \times W$ (лево) G-инвариантными векторными полями $\xi^{L}, \xi \in \mathfrak{m}$, дде $\xi^{L}(g, w)=\left(\xi^{l}(g), i P_{w}(\xi)\right)$. Больие того, $\operatorname{Ad} k \circ P_{w} \circ \operatorname{Ad} k^{-1}=P_{\operatorname{Ad} k(w)}$ на m для всех $w \in W, k \in K$.

ДокАЗАтЕльство. Определим вещественные подпространства $V_{1}, V_{2}$ пространства $T_{(e, w)}(G \times W)$, положив $V_{1}=\left\{(\xi, 0), \xi \in \mathfrak{m} \subset T_{e} G\right\}$ и $V_{2}=\{(0, u)$, $\left.u \in \mathfrak{m}=T_{w} W\right\}$. Отметим, что $\mathscr{P}(e, w) \subset V_{1}^{\mathbb{C}} \oplus V_{2}^{\mathbb{C}}$ и что пространства $\mathscr{P}(e, w)$, $V_{1}^{\mathbb{C}}, V_{2}^{\mathbb{C}}$ имеют одинаковую размерность.

Теперь, чтобы доказать сушествование отображения $P_{w}$, достаточно доказать, что $\mathscr{P}(e, w) \cap V_{k}^{\mathbb{C}}=0, k=1,2$. В силу равенства $(2.3) \widetilde{\Omega}\left(V_{k}, V_{k}\right)=0, k=1,2$ $\left([\mathfrak{m}, \mathfrak{m}] \subset \mathfrak{k}=\mathfrak{m}^{\perp}\right)$. Поэтому $\widetilde{\Omega}(Z, \bar{Z})=0$ для всех $Z \in \mathscr{P}(e, w) \cap V_{k}^{\mathbb{C}}$ и, как следует из предложения $2.5, Z \in\left(\mathscr{P} \cap \mathscr{K}_{W}^{\mathbb{C}}\right)(e, w)=0$. 
По определению (правого) действия $r_{k}$ группы Ли $K$ на $G \times W$ диффеоморфизм $r_{k *}$ отображает касательный вектор $\left(\xi^{l}(g), u\right)$ в точке $(g, w)$ в касательньй вектор $\left(\left(\operatorname{Ad}_{k^{-1}} \xi\right)^{l}(g k), \operatorname{Ad}_{k^{-1}} u\right)$ в точке $\left(g k, \operatorname{Ad}_{k-1} w\right)$, где $\xi \in \mathfrak{g}, w, u \in \mathfrak{m}, k \in K$. Тогда $\mathscr{P}-($ право $) K$-инвариантно тогда и только тогда, когда $\operatorname{Ad} k \circ P_{w} \circ \mathrm{Ad} k^{-1}=$ $P_{\mathrm{Ad} k(w)}$ на $\mathfrak{m}$.

ОПРЕДЕЛЕНИЕ 3.2. Пусть $W \subset \mathfrak{m}$ - открытое Ad $K$-инвариантное подмножество в $\mathfrak{m}$. Пусть $P: W \rightarrow \mathrm{GL}\left(\mathfrak{m}^{\mathbb{C}}\right), w \mapsto P_{w},-$ гладкое $K$-эквивариантное отображение, т.е. сплетающее присоединенное действие $\mathrm{Ad}$ группы $K$ на $W \subset \mathfrak{m}$ и действие $(k, B) \mapsto\left(\operatorname{Ad}_{\mathfrak{m}} k B \operatorname{Ad}_{\mathfrak{m}} k^{-1}\right)$ групшы $K$ на $\mathrm{GL}\left(\mathfrak{m}^{\mathbb{C}}\right):$

$\operatorname{Ad} k \circ P_{w} \circ \operatorname{Ad} k^{-1}=P_{\operatorname{Ad} k(w)}$ на $\mathfrak{m}$ для всех $w \in W, k \in K$.

Мы будем говорить, что (комплексные) распределения $\mathscr{F} \subset T^{\mathbb{C}}(G \times W)$ и $F \subset$ $T^{\mathbb{C}}(\Pi(G \times W))$ определены отображением $P$ (все $P_{w}$ невырождены), если

(1) $\mathscr{F}=\mathscr{K}_{W}^{\mathbb{C}}+\mathscr{P}$, где $\mathscr{P}$ порождается всюду ненулевыми на $G \times W$ (лево) $G$-инвариантными векторными полями $\xi^{L}, \xi \in \mathfrak{m}, \xi^{L}(g, w)=\left(\xi^{l}(g), i P_{w}(\xi)\right)$;

(2) $F=\Pi_{*}(\mathscr{F})$.

Такие (лево) $G$-инвариантные распределения $\mathscr{F}$ и $F$ мы будем обозначать через $\mathscr{F}(P)$ и $F(P)$ соответственно.

Мы будем исследовать комплексные структуры $F(P)$ (не обязательно кэлеровы) на $G$-инвариантных связных открытых подмножествах в $T(G / K)$. Зафиксируем базисы $\left\{X_{\beta}\right\}$ в k и $\left\{W_{b}\right\}$ в $\mathfrak{m}$. Пусть $\left\{w_{b}\right\}$ обозначает координаты вектора $w$ в $\mathfrak{m}$ относительно базиса $\left\{W_{b}\right\}$. Для произвольной вектор-функции $\tau: W \rightarrow \mathfrak{m}^{\mathbb{C}}$, $\tau=\sum_{b} \tau_{b}(w) W_{b}$, через $\vec{\tau}$ обозначим векторное поле

$$
\vec{\tau}(w) \stackrel{\text { def }}{=} \sum_{b} \tau_{b}(w) \frac{\partial}{\partial w_{b}} .
$$

Будем говорить, что векторное поле $Y \in \Gamma T(G \times W)$ горизонтально (вертикально), если $\operatorname{pr}_{v *}(Y)=0$ (соответственно $\left.\operatorname{pr}_{h *}(Y)=0\right)$, где $\operatorname{pr}_{v}: G \times W \rightarrow W$ (соответственно $\left.\operatorname{pr}_{h}: G \times W \rightarrow G\right)$ - обычная проекция.

Пусть $\sigma: T(G / K) \rightarrow T(G / K)$ - инволюция, которая отображает произвольный касательньй вектор $Y$ в точке $g K$ в вектор $-Y$ в этой же точке $g K$.

ПРЕДЛОЖЕНИЕ 3.3 [6]. Предположим, что $G / K$ - риманово симметрическое пространство. Пусть $\mathscr{F}=\mathscr{F}(P)$ - комплексное распределение на $G \times W$, определенное отображсением $P: W \rightarrow \mathrm{GL}\left(\mathfrak{m}^{\mathbb{C}}\right)$. Распределение $F(P)$ на $D$

(1) инволютивно тогда и только тогда, когда $\left[\xi^{L}, \eta^{L}\right]=[\xi, \eta]^{L} \in \Gamma \mathscr{K}_{W}^{\mathbb{C}}$, т.е. когда тохдество $[\overrightarrow{P(\xi)}, \overrightarrow{P(\eta)}](w)=-[w,[\xi, \eta]]$ выполняется на $W$ при всех $\xi, \eta \in \mathfrak{m}$

(2) лагранжево тогда и только тогда, когда для кажсдого $w \in W$ оператор $P_{w}$ симметричен относительно билинейной формы $\langle\cdot, \cdot\rangle$ на $\mathfrak{m}^{\mathbb{C}}$;

(3) является комплексной структурой тогда и только тогда, когда въполнено условие (1) и вещественная часть $\operatorname{Re} P_{w}$ линейного оператора $P_{w}$ невьрожсдена для всех $w \in W$, т.е. $\mathscr{F} \cap \overline{\mathscr{F}}=\mathscr{K}_{W}^{\mathbb{C}}$; 
(4) является кэлеровой структурой ( только тогда, когда выполнены условия (1), (2) и вещественная часть $\operatorname{Re} P_{w}$ (симметричного) линейного отображсения $P_{w}$ положстельно определена для всех $w \in W$, m.e. $\left\langle\left(\operatorname{Re} P_{w}\right)(\xi), \xi\right\rangle>0 \forall \xi \in \mathfrak{m}-\{0\}$.

Если $F(P)$ - комплексная структура и $W=-W$, то отображсние $\sigma$ является антиголоморфной инволючией тогда и только тогда, когда $\bar{P}_{w}=$ $P_{-w}$ для всех $w \in W$.

ДокАЗАТЕльство. Мы попытаемся объяснить смысл каждого условия этого предложения. В силу определения 3.2 распределение $\mathscr{P}$ (право) $K$-инвариантно. Поэтому распределение $\mathscr{K}_{W}$ (порожденное этим действием групшы Ли $K$ ) сохраняет (инвариантньм) распределение $\mathscr{F}:\left[\zeta_{W}^{L}, \mathscr{P}\right] \subset \mathscr{P}, \zeta \in \mathfrak{k}$.

Пусть $\xi, \eta \in \mathfrak{m}$. Так как векторные поля $\xi^{L}, \eta^{L}$ (лево) $G$-инвариантны, их горизонтальные и вертикальные компоненты не зависят от $w \in W$ и $g \in G$ соответственно:

$$
\xi^{L}(g, w)=\sum_{b} \xi_{b} W_{b}^{l}(g)+i \sum_{b}\left(P_{w}(\xi)\right)_{b} \frac{\partial}{\partial w_{b}} .
$$

Поэтому горизонтальная компонента векторного поля $\left[\xi^{L}, \eta^{L}\right]$ - это векторное поле $[\xi, \eta]^{l}$. C другой стороны, произвольное сечение распределения $\mathscr{F}$ определяется своей горизонтальной компонентой, а значит, из соотношений $[\mathfrak{k}, \mathfrak{m}] \subset \mathfrak{m}$ и $[\mathfrak{m}, \mathfrak{m}] \subset \mathfrak{k}$ следует, что $\left[\xi^{L}, \eta^{L}\right]-$ сечение распределения $\mathscr{F}(P)$ тогда и только тогда, когда выполнено условие (1) предложения 3.3 .

Так как $\mathscr{K}$ - ядро 2-формы $\widetilde{\Omega}$, то распределение $F(P)$ лагранжево тогда и только тогда, когда

$$
\widetilde{\Omega}_{(e, w)}\left(\left(\xi, i P_{w}(\xi)\right),\left(\eta, i P_{w}(\eta)\right)\right)=\left\langle\eta, i P_{w}(\xi)\right\rangle-\left\langle\xi, i P_{w}(\eta)\right\rangle-\langle w,[\xi, \eta]\rangle=0 .
$$

Но $\mathfrak{m} \perp[\mathfrak{m}, \mathfrak{m}]$ и, следовательно, $\left\langle\xi, i P_{w}(\eta)\right\rangle=\left\langle\eta, i P_{w}(\xi)\right\rangle$, т.е. мы пришли к условию (2). Используя аналогичные аргументы, получаем, что

$$
\begin{aligned}
-i \widetilde{\Omega}_{(e, w)}\left(\left(\xi+i \eta, i P_{w}(\xi+i \eta)\right),\left(\xi-i \eta,-i \bar{P}_{w}(\xi-i \eta)\right)\right) \\
=\left(\left\langle\left(\bar{P}_{w}+P_{w}\right)(\xi), \xi\right\rangle+\left\langle\left(\bar{P}_{w}+P_{w}\right)(\eta), \eta\right\rangle\right) \\
\quad+i\left(\left\langle\left(\bar{P}_{w}-P_{w}\right)(\xi), \eta\right\rangle-\left\langle\left(\bar{P}_{w}-P_{w}\right)(\eta), \xi\right\rangle\right) .
\end{aligned}
$$

В силу условия (2) предложения линейный оператор $\bar{P}_{w}-P_{w}$ симметричен. Поэтому поляризация $F(P)$ положительно определена тогда и только тогда, когда $\left\langle\left(\operatorname{Re} P_{w}\right)(\xi), \xi\right\rangle>0$ для всех $\xi \neq 0$ из $\mathfrak{m}$ (см. также предложение 2.5). Легко проверить, что условие (3) означает следующее: $\mathscr{F}+\overline{\mathscr{F}}=T^{\mathbb{C}}(G \times W)$. Таким образом, $F+\bar{F}=T^{\mathbb{C}} D$. Очевидно, что $\sigma_{*}(F(P))=\bar{F}(P)$ тогда и только тогда, когда $\bar{P}_{w}=P_{-w}$. Теперь предложение следует из леммы 2.4 и предложения 2.5.

3.2. Потенциальные функции. В этом пункте рассматривается класс $G$-инвариантных кэлеровых структур $F$ на касательном расслоении риманова симметрического пространства $G / K$, допускающих потенциальную функцию $f$ (т.е. $\Omega=-i \partial \bar{\partial} f)$ вида $q \circ \sqrt{H}$.

Пусть $H: T M \rightarrow \mathbb{R}$ - гамильтониан на $T M=T(G / K)$, ассоциированньй с данной метрикой $g: H(g K, Y)=g(Y, Y), Y \in T_{g K}(G / K)$. Обозначим через $\eta$ гамильтоново векторное поле функции $H / 2$. Векторное поле $2 \boldsymbol{\eta}(\Omega(2 \boldsymbol{\eta}, \cdot)=-d H)$ 
определяет на $T M$ геодезический поток. Чтобы упростить существенно все вычисления, мы, как и в предыдущем пункте, будем работать на тривиальном векторном расслоении $G \times \mathfrak{m}$. Для этого рассмотрим на $G \times \mathfrak{m}$ функцию $\widetilde{H}$ и векторное поле $\widetilde{\boldsymbol{\eta}}$, положив

$$
\widetilde{H}(g, w)=\langle w, w\rangle \text { и } \widetilde{\boldsymbol{\eta}}(g, w)=\left(w^{l}(g), 0\right) .
$$

Ясно, что $\widetilde{H}=\Pi^{*} H$. Так как билинейная форма $\langle\cdot, \cdot\rangle \operatorname{Ad} G$-инвариантна, то из (2.3) получим

$\widetilde{\Omega}_{(g, w)}\left(\widetilde{\boldsymbol{\eta}}(g, w),\left(\eta^{l}(g), u\right)\right)=-\langle w, u\rangle-\langle w,[w, \eta]\rangle=-\langle w, u\rangle=-\frac{1}{2} d \widetilde{H}_{(g, w)}\left(\eta^{l}(g), u\right)$,

где $\eta \in \mathfrak{g}, u \in \mathfrak{m}=T_{w} \mathfrak{m}$. Таким образом, $\Pi^{*} \Omega(\widetilde{\boldsymbol{\eta}}, \cdot)=-d \widetilde{H} / 2=-\Pi^{*} d H / 2$. Но форма $\Omega$ невырождена, поэтому $\Pi_{*} \widetilde{\boldsymbol{\eta}}-$ корректно определенное векторное поле и $\Pi_{*} \widetilde{\boldsymbol{\eta}}=\boldsymbol{\eta}$ по определению.

Пусть $\Xi$ и $\widetilde{\Xi}-$ векторные поля на $T(G / K)$ и $G \times \mathfrak{m}$ соответственно, определенные тождествами

$$
\imath(\Xi) \Omega=\theta \quad \text { и } \widetilde{\Xi}(g, w)=(0, w) .
$$

В силу соотношений $(2.2),(2.3) \imath(\widetilde{\Xi}) \widetilde{\Omega}=\widetilde{\theta}$. Учитывая, что векторное поле $\widetilde{\Xi}$ (право) $K$-инвариантно, мы получаем, что $\Pi_{*}(\widetilde{\Xi})=\Xi$. В этом пункте мы введем в рассмотрение векторное поле, определенное на $G \times W$, которое сыграет существенную роль в дальнейшем изложении. Пусть $\lambda: r(W) \rightarrow \mathbb{C}$ - гладкая функция. Эта функция определяет $\operatorname{Ad} K$-инвариантную функцию $\lambda: w \mapsto \lambda(r(w))$ на $W$ и единственную $G$-инвариантную функцию на $G \times W$ и $(G \times W)$. Для упрошения обозначений мы часто будем писать $\lambda(r)$ вместо $\lambda \circ \sqrt{H}$ и $\lambda \circ \sqrt{\widetilde{H}}, \widetilde{\boldsymbol{\eta}}$ и $\widetilde{\Xi}(\boldsymbol{\eta}$ и $\Xi)$ вместо их ограничений на $G \times W(\Pi(G \times W))$. Рассмотрим теперь векторное поле $\Upsilon^{\lambda}$ на $G \times W$, заданное равенством $\Upsilon^{\lambda}(g, w)=\left(w^{l}(g), i \lambda(|w|) w\right)$. Ясно, что $\Upsilon^{\lambda}=\widetilde{\eta}+i \lambda \widetilde{\Xi}$.

ПРЕДЛОЖЕНИЕ 3.4. Пусть $F-G$-инвариантная кәлерова структура на $G$-инвариантной области $D$ в выколотом касательном расслоении риманова симметрического пространства $G / K$. Существует вещественная гладкая функиия $q: r(W) \rightarrow \mathbb{R}$ такая, что $\theta=\operatorname{Im} \bar{\partial}(q \circ \sqrt{H})$ тогда и только тогда, когда векторное поле $\boldsymbol{\eta}+i \lambda(r) \Xi$, где $\lambda(r)=2 r / q^{\prime}(r)$, является сечением распределения $F=F(P)$. В этом случае каждый вектор $w \in W-$ собственный вектор оператора $P_{w}$ с собственным значением $\lambda(|w|)$, а $\lambda$ - положсительная функиия.

ДокАЗАТЕЛЬСтво. Необходимость условий предложения следует из результатов работы [9; дополнение A]. Для полноты изложения и, главным образом, чтобы зафиксировать обозначения, мы докажем это здесь. Так как симплектическая форма $\Omega J$-инвариантна, то для каждого $Y \in \Gamma T D$ выполняется цепочка равенств

$$
\begin{aligned}
\Omega(J \boldsymbol{\eta}, Y) & =\Omega(\boldsymbol{\eta},-J Y)=\lambda \frac{1}{2} d q(J Y)=\lambda \frac{1}{2}(\bar{\partial} q(-i Y)+\partial q(i Y)) \\
& =\lambda \operatorname{Im} \bar{\partial} q(Y)=\lambda \theta(Y)=\Omega(\lambda \Xi, Y) .
\end{aligned}
$$

Следовательно, $J \boldsymbol{\eta}=\lambda \Xi$. Для доказательства достаточности мы отметим следуюшее: $\boldsymbol{\eta}+i \lambda \Xi \in \Gamma F$ тогда и только тогда, когда $\widetilde{\boldsymbol{\eta}}+i \lambda \widetilde{\Xi}$ есть сечение распределения 
$\mathscr{F}=\mathscr{F}(P)$ на $\Pi^{-1}(D)$, т.е. ког да каждый вектор $w \in W$ - собственный вектор оператора $P_{w}$ с собственным значением $\lambda(|w|)$. В частности, отсюда вытекает то, что для нас сушественно в этом месте доказательства и что мы сформулируем в виде леммы.

ЛЕмма 3.5. Пусть $\lambda: r(W) \rightarrow \mathbb{C}-$ гладкая функиия $с$ положсительной вещественной частью $\left(W \subset \mathfrak{m}^{0}\right)$. Если $\Upsilon^{\lambda} \in \Gamma \mathscr{F}$, то функиия $q \circ \sqrt{H}$, где

$$
q(r)=\int r \cdot \frac{\lambda(r)+\bar{\lambda}(r)}{|\lambda|^{2}(r)} d r
$$

является потенциальной функиией для кәлеровой структуры $F$.

По определению $\bar{\partial}(q \circ \sqrt{H})|F=d(q \circ \sqrt{H})| F$ и $\bar{\partial}(q \circ \sqrt{H}) \mid \bar{F}=0$. Обозначим через $\Delta$ 1-форму $\Pi^{*}(\bar{\partial}(q \circ \sqrt{H}))$ на $G \times W$. Тогда для каждого $\xi \in \mathfrak{m}, \zeta \in \mathfrak{k}, w \neq 0$ : $\Delta_{(g, w)}\left(\xi^{l}(g),-i \bar{P}_{w}(\xi)\right)=0, \Delta_{(g, w)}\left(\zeta^{l}(g),[w, \zeta]\right)=0$ и

$$
\Delta_{(g, w)}\left(\xi^{l}(g), i P_{w}(\xi)\right)=r^{-1} q^{\prime}\left\langle w, i P_{w}(\xi)\right\rangle=i r^{-1} q^{\prime} \lambda\langle w, \xi\rangle
$$

так как оператор $P_{w}$ симметричен относительно формы $\langle\cdot, \cdot\rangle$. Теперь, используя инвариантность пространства $\langle w\rangle^{\perp}$ относительно $P_{w}$, мы получаем, что $\Delta_{(e, w)}\left(\xi_{0}, 0\right)=0, \Delta_{(e, w)}\left(0, \xi_{0}\right)=0$ для всех $\xi_{0} \in\langle w\rangle^{\perp} \subset \mathfrak{m}$. Так как $[w, \zeta] \perp w$ для всех $\zeta \in \mathfrak{k}$, то $\Delta_{(e, w)}(\zeta, 0)=0$. Следовательно,

$$
\Delta_{(e, w)}(w,-i \bar{\lambda} w)=0, \quad \Delta_{(e, w)}(w, i \lambda w)=i r^{-1} q^{\prime} \lambda\langle w, w\rangle
$$

Таким образом, для произвольных $\eta \in \mathfrak{g}, u \in \mathfrak{m}$ мы имеем

$$
\begin{aligned}
\Delta_{(e, w)}(\eta, u) & =\frac{q^{\prime}}{|w|(\lambda+\bar{\lambda})}\left(i|\lambda|^{2}\langle w, \eta\rangle+\lambda\langle w, u\rangle\right) \\
& =\frac{q^{\prime}}{|w|(\lambda+\bar{\lambda})}\left(i|\lambda|^{2} \widetilde{\theta}+\frac{1}{2} \lambda d \widetilde{H}\right)_{(e, w)}(\eta, u) .
\end{aligned}
$$

Поэтому функция $q \circ \sqrt{H}$ является потенциалом для $F$, если $q^{\prime}|\lambda|^{2} /(|w|(\lambda+\bar{\lambda})) \equiv 1$. В этом случае, если дополнительно предположить вещественность функции $\lambda$, мы получим требуемое свойство $\operatorname{Im} \Pi^{*}(\bar{\partial} q)=\widetilde{\theta}=\Pi^{*} \theta$.

Следующие свойства отображения $P$ нам необходимы для доказательства теоремы 4.14 .

ПРЕДЛОЖЕНИЕ 3.6. Пусть $M=G / K$ - риманово симметрическое пространство и $\mathscr{F}=\mathscr{F}(P)$ - комплексное распределение на $G \times W, W \subset \mathfrak{m}^{0}$, определенное отображением $P: W \rightarrow \mathrm{GL}\left(\mathfrak{m}^{\mathbb{C}}\right)$. Предположим, что векторное поле $\Upsilon^{\lambda}$ является сечением $\mathscr{F}(P)$. Если распределение $F(P)$ инволютивно и лагранжсево, то

$$
-\operatorname{ad}^{2} w \mid \mathfrak{m}-P_{w}^{2}+\lambda(r)\left(P_{w}-\left.\frac{d}{d t}\right|_{0} P_{w+t w}+\frac{d \lambda}{d r} r^{-1} w \otimes w\right)=0 \quad \forall w \in W
$$

$2 \partial e(w \otimes w)(\xi) \stackrel{\text { def }}{=}\langle w, \xi\rangle w, \xi \in \mathfrak{m}$. 
ДокаЗАТЕЛЬСтво. Нам необходимо вычислить коммутатор $Y=\left[\Upsilon^{\lambda}, \xi^{L}\right]$, $\xi \in \mathfrak{m}$. Используя обозначения доказательства предложения 3.3 , мы получаем:

$$
\begin{aligned}
Y= & {\left[w_{b} W_{b}^{l}(g)+i \lambda(r) w_{b} \frac{\partial}{\partial w_{b}}, \xi_{b} W_{b}^{l}(g)+i\left(P_{w}(\xi)\right)_{b} \frac{\partial}{\partial w_{b}}\right] } \\
= & {[w, \xi]_{\beta} X_{\beta}^{l}(g)-i\left(P_{w}(\xi)\right)_{b} W_{b}^{l}(g) } \\
& +\left(\lambda(r) P_{w}(\xi)+\frac{d \lambda}{d r} r^{-1}\left\langle w, P_{w}(\xi)\right\rangle w-\left.\lambda(r) \frac{d}{d t}\right|_{0} P_{w+t w}(\xi)\right)_{b} \frac{\partial}{\partial w_{b}} .
\end{aligned}
$$

Так как произвольное сечение распределения $\mathscr{F}(P)$ определяется своей горизонтальной компонентой и оператор $P_{w}$ симметричен, векторное поле $Y$ есть сечение распределения $\mathscr{F}(P)$ только в том случае, когда выполнено условие $(3.2)\left(P_{w}(w)=\right.$ $\lambda(|w|) w)$.

3.3. Кэлеровы структуры и локальные диффеоморфизмы. В этом пункте мы, обобщая конструкцию работы [5], построим “группу" локальных диффеоморфизмов и изучим ее действие на пространстве локальных $G$-инвариантных кэлеровых структур $\{(F(P), \Pi(G \times W))\}$ на $T(G / K)$, где $G / K$ - риманово симметрическое пространство.

Для этого мы рассмотрим диффеоморфизм $\Psi(\gamma)$, отображающий открытое множество $U_{\gamma}=G \times W_{\gamma}, W_{\gamma} \subset \mathfrak{m}^{0}$, на множество $U_{\delta}=G \times W_{\delta}$ и заданньй формулой

$$
\Psi(\gamma): G \times W_{\gamma} \rightarrow G \times W_{\delta}, \quad \Psi(\gamma)(g, w)=(g, \gamma(|w|) w),
$$

где $\gamma: r\left(W_{\gamma}\right) \rightarrow \mathbb{R}^{+}$- гладкая положительная функция такая, что $\frac{d}{d r}(r \gamma(r))>0$. Обратный диффеоморфизм $\Psi^{-1}(\gamma)$ - это отображение $\Psi(\delta): U_{\delta} \rightarrow U_{\gamma}(3.3)$, где $\delta:(r \gamma)\left(W_{\gamma}\right) \rightarrow \mathbb{R}^{+}$- единственная функция, определяемая уравнением $\gamma(r \delta(r)) \cdot \delta(r) \equiv 1$. Нетрудно показать, что $\frac{d}{d r}(r \delta(r))>0$.

Образ $\Psi(\gamma)_{*} \xi^{L}$ векторного поля $\xi^{L} \mid U_{\gamma}, \xi^{L}(g, w)=\left(\xi^{l}(g), i P_{w}(\xi)\right), W_{\gamma}=W$, есть векторное поле

$$
\left(\Psi(\gamma)_{*} \xi^{L}\right)_{(g, w)}=\left(\xi^{l}(g), i \gamma(r \delta(r)) \cdot P_{\delta(r) w}(\xi)+i\left(\gamma^{\prime}(r \delta(r)) \delta(r) r^{-1}\right)\left\langle w, P_{\delta(r) w}(\xi)\right\rangle \cdot w\right),
$$

где $r=|w|, w \in W_{\delta}$. Это означает, что образ $\Psi(\gamma)_{*}(\mathscr{F}(P))$ - это распределение $\mathscr{F}\left(P^{\gamma}\right)$ на $G \times W_{\delta}$ с

$$
P_{w}^{\gamma}=\frac{1}{\delta(r)} P_{\delta(r) w}+\left(\frac{1}{(r \delta(r))^{\prime}}-\frac{1}{\delta(r)}\right) r^{-2} \cdot w \otimes\left(P_{\delta(r) w}(w)\right), \quad w \in W_{\delta},
$$

где $r=|w|$. Но диффеоморфизм $\Psi(\gamma)$ коммутирует с (левым) $G$-действием и (правым) $K$-действием на $G \times \mathfrak{m}$, а значит, индуцирует диффеоморфизм из $D_{\gamma}=\Pi\left(G \times W_{\gamma}\right)$ на $D_{\delta}=\Pi\left(G \times W_{\delta}\right)$. Этот диффеоморфизм мы обозначим тоже через $\Psi(\gamma)$. Ясно, что $\Psi(\gamma)_{*}(F(P))$ - комплексная структура. Оператор $P_{w}^{\gamma}$ симметричен тогда и только тогда, когда $\delta(r) w \in W_{\gamma}$ является собственным вектором оператора $P_{\delta(r) w}$. Из предложения 3.3 в этом случае следует, что $P_{w}^{\gamma}$ определяет кэлерову структуру на $D_{\delta} \subset T(G / K)$. Итак, доказана 
Лемма 3.7. Пусть $F(P)$ - кәлерова структура на $D_{\gamma}=\Pi\left(G \times W_{\gamma}\right)$. Kомnлексная структура $\Psi(\gamma)_{*}(F(P))$ на $D_{\delta} \subset T(G / K)$ кәлерова тогда и только тогда, когда каждый $w \in W_{\gamma}$ является собственным вектором оператора $P_{w}$. Эта структура совпадает с распределением $F\left(P^{\gamma}\right)$, где $P^{\gamma}$ имеет вид (3.4), на $D_{\delta}$.

3.4. Каноническая кэлерова структура на касательном расслоении компактного симметрического пространства. В этом пункте $G$ - компактная связная группа Ли. Пусть $G^{\mathbb{C}}$ и $K^{\mathbb{C}}$ - комплексификации групп Ли $G$ и $K$ соответственно. Так как группа Ли $G$ компактна, то топологически $K^{\mathbb{C}}=K \exp (i \mathfrak{k})$ и $G^{\mathbb{C}}=G \exp (i \mathfrak{m}) \exp (i \mathfrak{k})[10 ;$ теорема 4$]$, т.е. отображения

$$
\begin{gathered}
G \times \mathfrak{m} \times \mathfrak{k} \rightarrow G^{\mathbb{C}}, \quad(g, w, \zeta) \mapsto g \exp (i w) \exp (i \zeta), \\
K \times \mathfrak{k} \rightarrow K^{\mathbb{C}}, \quad(k, \zeta) \mapsto k \cdot \exp (i \zeta),
\end{gathered}
$$

являются диффеоморфизмами. Тогда отображение

$$
G \times_{K} \mathfrak{m} \rightarrow G^{\mathbb{C}} / K^{\mathbb{C}}, \quad[(g, w)] \mapsto g \exp (i w) K^{\mathbb{C}}
$$

- $G$-эквивариантный диффеоморфизм. Это отображение определяет на многообразии $T(G / K)$ комплексную структуру $F^{c}$.

Лемма 3.8. Пусть $G$ - компактная связная группа Ли. Комплексная структура $F^{c}$ на касательном расслоении симметрического пространства $G / K$ совпадает с распределением $F\left(P^{c}\right)$, где

$$
P_{w}^{c}: \mathfrak{m} \rightarrow \mathfrak{m}, \quad P_{w}^{c}=\left.\frac{\operatorname{ad} w \cos (\operatorname{ad} w)}{\sin (\operatorname{ad} w)}\right|_{\mathfrak{m}}, \quad w \in \mathfrak{m}
$$

Комплексная структура $F^{c}$ кәлерова.

ДокАЗАТЕЛЬСтво формулы (3.7), основанное на процедуре редукции, можно найти в работе [6]. Здесь мы докажем лемму, используя диффеоморфизм (3.5) и $G$-инвариантность $F^{c}$. Пусть $\mathscr{F}^{c}=\Pi_{*}^{-1}\left(F^{c}\right)$. Групу $G^{\mathbb{C}}$, рассматриваемую как вешественную группу Ли, обозначим через $G^{\mathbb{R}}$. Каноническая комплексная структура $F^{c}$ на $G^{\mathbb{R}}$ определяется (лево) $G^{\mathbb{R}}$-инвариантными $(0,1)$-векторньми полями $\xi^{l}+i(I \xi)^{l}$, где $\xi \in \mathfrak{g}$ и $I$ - комплексная структура на $\mathfrak{g}^{\mathbb{C}}$. Поэтому чтобы найти $\mathscr{F}^{c}=\Pi_{*}^{-1}\left(F^{c}\right)$, нужно вычислить образ $G^{\mathbb{R}}$-инвариантного векторного поля $\xi^{l}+i(I \xi)^{l}, \xi \in \mathfrak{g}$, при отображении $G^{\mathbb{C}} \rightarrow G \times_{K} \mathfrak{m}, g \exp (i w) \exp (i \zeta) \mapsto[g, w]$. В силу формул (3.5) $\exp (i w) \exp (t \xi)=g(t) \exp (i v(t)) \exp (i k(t))$, где $g_{0}=e, v_{0}=w$ и $k_{0}=0$. Тогда из уравнения

$$
\exp t \xi=(\exp (-i w) g(t) \exp i w)(\exp (-i w) \exp i v(t)) \exp (i k(t)), \quad \xi \in \mathfrak{m}
$$

мы получим два вещественных уравнения для касательных векторов $g_{0}^{\prime} \in \mathfrak{g}$, $v_{0}^{\prime} \in \mathfrak{m}, k_{0}^{\prime} \in \mathfrak{k}:$

$$
\xi=\exp (-i \operatorname{ad} w)\left(g_{0}^{\prime}\right)+\frac{1-\exp (-i \operatorname{ad} w)}{\operatorname{ad} w}\left(v_{0}^{\prime}\right)+i k_{0}^{\prime}
$$


Приравнивая компоненты, лежащие в подпространствах $\mathfrak{m}$ и ḱ соответственно, получаем $g_{0}^{\prime}=\cos ^{-1}(\operatorname{ad} w)(\xi) \in \mathfrak{m}, v_{0}^{\prime}=0$.

Заменяя в (3.8) $\xi \mapsto i \xi$ и решая опять те же два уравнения, мы получаем выражения $\widehat{g}_{0}^{\prime}=\frac{\cos (\operatorname{ad} w)-1}{\sin (\operatorname{ad} w)} \xi \in \mathfrak{k}, \widehat{v}_{0}^{\prime}=\frac{\operatorname{ad} w \cos (\operatorname{ad} w)}{\sin (\operatorname{ad} w)} \xi$. Учитьвая, что $\left(i \widehat{g}_{0}^{\prime}, i\left[w, \widehat{g}_{0}^{\prime}\right]\right)$ является $\mathscr{K}^{\mathbb{C}}(e, w)$-компонентой вектора $\left(g_{0}^{\prime}+i \widehat{g}_{0}^{\prime}, i \widehat{v}_{0}^{\prime}\right)$, мы находим его $\mathscr{P}(e, w)$-компоненту, определяемую (3.7). Из предложения 3.3 следует теперь, что $P^{c}$ определяет кэлерову структуру на $T(G / K)$.

Следствие из леммы 3.8 выделяет каноническую комплексную структуру $F^{c}$ в множестве всех комплексных структур на $\sigma$-инвариантных окрестностях $U$, содержащих нулевое сечение $G / K \subset T(G / K)$.

СлЕДСТВИЕ 3.8.1. Пусть $F$ - комплексная структура на б-инвариантной области $U \subset T(G / K)$, содержащей $G / K$, обладающая свойствами:

(1) $\sigma_{*}(F)=\bar{F}$

(2) $\operatorname{Im} \bar{\partial} H=\theta$.

Tогда $F=F^{c} \mid U$.

ДокаЗАтельство. Так как $\bar{P}_{w}^{c}=P_{-w}^{c}$ для всех $w \in \mathfrak{m}$, то из предложения 3.3 вытекает, что $\sigma_{*}\left(F^{c}\right)=\overline{F^{c}}$. Теперь, применяя предложение 3.4 к структуре $F^{c}$, получаем второе условие: $\operatorname{Im} \bar{\partial} H=\theta$ (относительно $F^{c}$ ). Но теорема Гийемина и Стензеля [11] утверждает, что комплексная структура $F$ на множестве $U$, содержашем $G / K$, со свойствами $(1),(2)$ единственна. Поэтому $F=F^{c} \mid U$.

ЗАмечаниЕ 3.9. Другое доказательство формулы (3.7) может быть получено с использованием выражений (3)-(5) работы [12] для адаптированной структуры $F^{A}$ на $T(G / K)$ в терминах геометрических структур, ассоциированных с метрикой $g$ на $G / K$. Структуры $F^{c}$ и $F^{A}$ совпадают [13].

Теперь, используя структуру $F^{c}$ и результаты предыдущих пунктов, мы построим новое семейство $G$-инвариантных кэлеровых структур на выколотых касательных расслоениях $T^{0}(G / K)=T(G / K)-G / K$ и соответствуюшее семейство распределений на $G \times \mathfrak{m}^{0}$, где $\mathfrak{m}^{0}=\mathfrak{m}-\{0\}$.

Мы продолжим изложение с прежними обозначениями, положив $P=P^{c}(3.7)$. Так как $P_{w}^{c}(w)=w$, то в силу леммы 3.7 отображение $P^{\gamma}: w \mapsto P_{w}^{\gamma}(3.4)$ определяет кэлерову структуру в области $D_{\delta}$ и

$$
P_{w}^{\gamma}=\left.\frac{1}{\delta} \cdot \frac{\operatorname{ad}(\delta w) \cos (\operatorname{ad} \delta w)}{\sin (\operatorname{ad} \delta w)}\right|_{\mathfrak{m}}+\left(\frac{1}{(r \delta)^{\prime}}-\frac{1}{\delta}\right) r^{-2} \cdot w \otimes w, \quad w \in W_{\delta}
$$

где $r=|w|, \delta=\delta(r)$.

Пусть $\lambda: \mathbb{R}^{+} \rightarrow \mathbb{R}^{+}$- гладкая положительная функция, а функция $a$ определяется равенством

$$
\frac{d}{d r} \ln a(r)=\frac{1}{\lambda(r)}
$$

Положим $\delta_{\varepsilon}(r)=r^{-1} \ln (a(r) / \varepsilon), \varepsilon \in \mathbb{R}^{+}$. Так как $\left(r \delta_{\varepsilon}(r)\right)^{\prime}=a^{\prime}(r) / a(r)$, то собственное значение $\lambda_{\varepsilon}(r)=a(r) / a^{\prime}(r)$ эндоморфизма $P_{w}^{\gamma_{\varepsilon}}$, соответствующее 
вектору $w$, не зависит от $\varepsilon$. Как легко следует из $(3.9)$, для произвольного $\xi \in \mathfrak{m}$ сушествует граничное векторное поле $\xi_{0}^{L}=\lim _{\varepsilon \rightarrow 0} \Psi\left(\gamma_{\varepsilon}\right)_{*} \xi^{L}$ на $G \times \mathfrak{m}^{0}$ :

$\lim _{\varepsilon \rightarrow 0} \Psi\left(\gamma_{\varepsilon}\right)_{*} \xi^{L}(g, w)=\left(\xi^{l}(g), i \sqrt{-\operatorname{ad}^{2} w}(\xi)+i\left(\frac{\lambda(r)}{r^{2}}\right)\langle w, \xi\rangle w\right), \quad r=|w|, w \in \mathfrak{m}^{0}$.

Это векторное поле гладко на открытом подмножестве $G \times R(\mathfrak{m})$, где $R(\mathfrak{m})$ - множество регулярных элементов из $\mathfrak{m}$. В силу утверждения (1) предложения 3.3 и леммы $3.7\left[\Psi\left(\gamma_{\varepsilon}\right)_{*} \xi^{L}, \Psi\left(\gamma_{\varepsilon}\right)_{*} \eta^{L}\right]=[\xi, \eta]^{L}$ и, следовательно, $\left[\xi_{0}^{L}, \eta_{0}^{L}\right]=[\xi, \eta]^{L}$ на $G \times R(\mathfrak{m})$. В самом деле, $\operatorname{ad} w \operatorname{ctg}(\operatorname{ad} w) \mid \mathfrak{m}=\widehat{w} \operatorname{cth} \widehat{w}$, где $\widehat{w}=\sqrt{-\operatorname{ad}^{2} w} \mid \mathfrak{m}$. Поэтому если $[u, w]=0, u \in \mathfrak{m}$, то для $w_{t}=w+t u$ :

$$
\left.\frac{d}{d t}\right|_{t=0} \operatorname{cth}\left(\delta_{\varepsilon}\left(\left|w_{t}\right|\right) \widehat{w}_{t}\right)=-\operatorname{sh}^{-2}\left(\delta_{\varepsilon}(|w|) \widehat{w}\right) \cdot O\left(\delta_{\varepsilon}(|w|)\right) \text { при } \varepsilon \rightarrow 0 ;
$$

если $w_{t}=\operatorname{Ad} k_{t}(w)$, то

$$
\left.\frac{d}{d t}\right|_{t=0} \operatorname{Ad} k_{t} \cdot \operatorname{cth}\left(\delta_{\varepsilon}(|w|) \widehat{w}\right) \operatorname{Ad} k_{t}^{-1}=\left[\operatorname{ad} \zeta, \operatorname{cth}\left(\delta_{\varepsilon}(|w|) \widehat{w}\right)\right],
$$

где $k_{t}=\exp t \zeta, \zeta \in \mathfrak{k}$. Таким образом,

$$
\lim _{\varepsilon \rightarrow 0}\left(\left.\frac{d}{d t}\right|_{t=0} P_{w+t u}^{\gamma_{\varepsilon}}\right)=\left.\frac{d}{d t}\right|_{t=0}\left(\lim _{\varepsilon \rightarrow 0} P_{w+t u}^{\gamma_{\varepsilon}}\right)
$$

для произвольных $w \in R(\mathfrak{m})$ и $u \in \mathfrak{m}\left(\delta_{\varepsilon}^{\prime}(r)=O\left(\delta_{\varepsilon}(r)\right)\right.$ при $\varepsilon \rightarrow 0, \operatorname{ker}(\operatorname{ad} w \mid \mathfrak{m})=$ $\left.\operatorname{ad} w(\mathfrak{k})^{\perp} \subset \mathfrak{m}\right)$.

Если $G / K$ - симметрическое пространство ранга один, то $R(\mathfrak{m})=\mathfrak{m}^{0}$ и ядро оператора $\operatorname{ad}^{2} w \mid \mathfrak{m}, w \in \mathfrak{m}^{0}$, порождается вектором $w$. Поэтому отображение $\mathfrak{m}^{0} \rightarrow \mathrm{GL}(\mathfrak{m}), w \mapsto P_{w}$, заданное формулой

$$
P_{w}=\sqrt{-\operatorname{ad}^{2} w} \mid \mathfrak{m}+\frac{\lambda(|w|)}{|w|^{2}} \cdot w \otimes w, \quad w \in \mathfrak{m}^{0},
$$

определяет кэлерову структуру на $T^{0}(G / K)$. Мы доказали

ПРЕДЛОЖЕНИЕ 3.10. Пусть $G / K$ - компактное симметрическое пространство ранга один. Для произвольной гладкой положсительной функиии $\lambda: \mathbb{R}^{+} \rightarrow \mathbb{R}^{+}$отобрахсение $P: \mathfrak{m}^{0} \rightarrow \mathrm{GL}(\mathfrak{m})$ (3.10) определяет кәлерову структуру на $T^{0}(G / K)$.

Отметим, что структура $F(P)$, определяемая $P(3.10)$, где $\lambda(\mathbf{r})=\mathbf{r}$, совпадает со структурой $J_{S}[5]$. Мы можем дополнить предложение 3.10 следствием.

СЛЕДСТВИЕ 3.10.1. Пусть $\mathfrak{g}$ - компактная полупростая алгебра Ли и $\mathfrak{k}-$

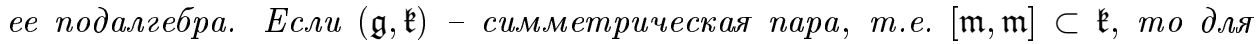
произвольных векторов $\xi, \eta \in \mathfrak{m}$ коммутационное соотношение

$\left[\left(\sqrt{-\operatorname{ad}^{2} w}(\xi)\right)_{b} \frac{\partial}{\partial w_{b}},\left(\sqrt{-\operatorname{ad}^{2} w}(\eta)\right)_{b} \frac{\partial}{\partial w_{b}}\right]=-[w,[\xi, \eta]]_{b} \frac{\partial}{\partial w_{b}}, \quad w \in R(\mathfrak{m})$,

выполнено на множестве регулярных точек $R(\mathfrak{m})$.

ДокАЗАТЕЛЬство. Чтобы доказать следствие, достаточно использовать предельный переход, как было показано выше, но для функции $\lambda(r)=\varepsilon>0$ $\left(\frac{d}{d r} \ln a(r)=\varepsilon^{-1}\right)$. Тогда $\delta_{\varepsilon}(r) \rightarrow \infty$ и $\delta_{\varepsilon}^{\prime}(r)=O\left(\delta_{\varepsilon}(r)\right)$ при $\varepsilon \rightarrow 0$. 


\section{§4. Инвариантные кэлеровы структуры на инвариантных областях касательных расслоений симметрических пространств ранга один}

Мы продолжим изложение, используя обозначения предыдущих параграфов, но здесь, если не оговорено противное, предположим, что $G / K$ - симметрическое пространство ранга один с полупростой связной группой Ли $G$ и связной компактной подгруппой $K$. Чтобы согласовать описания кэлеровых структур для симметрических пространств классических групп Ли $G$, мы выберем в качестве положительно определенного скалярного произведения на $\mathfrak{m}$ ограничение нормализованной формы $-\frac{\varepsilon}{2} \operatorname{Tr}($ на $\mathfrak{g})$, ассоциированной со стандартным представлением алгебры Ли $\mathfrak{g}(\varepsilon=1$ для компактных и $\varepsilon=-1$ для некомпактных симметрических пространств). Это скалярное произведение для классических симметрических пространств мы обозначим через $\langle\langle\cdot, \cdot\rangle\rangle$ и положим $\mathbf{r}(w)=\|w\|=\sqrt{\langle\langle w, w\rangle}$.

Пусть $S\left(\mathfrak{m}^{\mathbb{C}}\right)$ - множество всех обратимых симметричных операторов из $\mathrm{GL}\left(\mathfrak{m}^{\mathbb{C}}\right)$. Обозначим через $\mathfrak{K}(D)=\mathfrak{K}(W)$ множество всех $G$-инвариантных кэлеровых структур (с кэлеровой формой $\Omega$ ) на $G$-инвариантных областях $D=\Pi(G \times W), W=$ $\operatorname{Ad} K(W)$ в $T(G / K)$. Здесь мы опишем множество $\mathfrak{K}(W)$, т.е. множество всех $K$-эквивариантных отображений $P: W \rightarrow S\left(\mathfrak{m}^{\mathbb{C}}\right), w \mapsto P_{w}($ см. (3.1)), для которых выполнены условия предложения 3.3. Для этого мы рассмотрим более детально представление $R_{\mathfrak{m}}: K \rightarrow \mathrm{GL}(\mathfrak{m}), k \mapsto \operatorname{Ad} k \mid \mathfrak{m}$, групшы Ли $K$ в пространстве т и его ограничение на стационарную подгруппу $K_{w}=\{k \in K: \operatorname{Ad} k(w)=w\}$ элемента $w \in W$. В силу уравнения (3.1) оператор $P_{w}$ коммутирует со всеми операторами $\operatorname{Ad} k \mid \mathfrak{m}, k \in K_{w}$ :

$$
\operatorname{Ad} k \cdot P_{w}=P_{w} \cdot \operatorname{Ad} k \text { на } \mathfrak{m} \text { для всех } w \in W, k \in K_{w} .
$$

Так как $G / K$ - симметрическое пространство ранга один, то каждая $\mathrm{Ad} K$-инвариантная функция на $\mathfrak{m}$ является функцией от $r(w)=|w|$ и для произвольных ненулевых векторов $w, u \in W$ стационарные подгруппы $K_{w}$ и $K_{u}$ сопряжены [14] $(w$ порождает подпространство Картана в $\mathfrak{m})$. В частности, $W=r^{-1}(O)$, где $O$ - открытое связное подмножество в $[0,+\infty)$. Обозначим через $W^{0}$ подмножество $W-\{0\}$. Положим $D^{0}=D-G / K$.

ЗАмЕчАНИЕ 4.1. Пусть $\mathfrak{a}$ - одномерное подпространство Картана в $\mathfrak{m}$, содержащее элемент $w, \mathfrak{t}$ - подалгебра Картана полупростой алгебры Ли $\mathfrak{g}$, содержащая $\mathfrak{a}$. Обозначим через $\Sigma$ ограниченную систему корней, ассоциированную с парой $(\mathfrak{a}, \mathfrak{t}) ;$ для корня $\sigma \in \Sigma$ пусть $m_{\sigma}$ обозначает его кратность [14; гл. IX, $\left.\S 6\right]$. Тогда существует корневое разложение $\mathfrak{m}=\mathfrak{a} \oplus \sum_{\sigma \in \Sigma^{+}} V^{\sigma}$ пространства $\mathfrak{m}$, где $\Sigma^{+}-$множество положительных ограниченных корней и подпространства $V^{\sigma}$ - собственные подпространства линейного отображения $\operatorname{ad}^{2} h, h \in \mathfrak{a}$, размерности $m_{\sigma}$ с собственными значениями $\sigma^{2}(h)$ [14; гл. VII, лемма $\left.2.3 ; \S 10\right]$. Отметим, что величины $\sigma(h)$ чисто мнимые, если $(\mathfrak{g}, \mathfrak{k})$ - симметрическая пара компактного типа, и вешественные в некомпактном случае. Ясно, что это разложение $\operatorname{Ad} K_{w}$-инвариантно. В нашем случае ранга один $\Sigma=\{ \pm \sigma\}$ для симметрического пространства $G / K$ $\mathrm{c}(\mathfrak{g}, \mathfrak{k}) \simeq(\operatorname{so}(n+1), \mathrm{so}(n))$ и дуального к нему некомпактного $\operatorname{SO}_{0}(1, n) / \mathrm{SO}(n)$; $\Sigma=\{ \pm \sigma, \pm 2 \sigma\}$ для всех остальных симметрических пространств ранга один [14; таблица VI]. Больше того, $m_{2 \sigma}=1$ для комплексных проективных пространств $\mathbb{C} P^{n}, n \geqslant 2$, и $m_{2 \sigma}=3$ для кватернионных проективных пространств $\mathbb{H} P^{n}, n \geqslant 2$. 
Замечание 4.2. Предположим, что полупростая группа Ли $G$ компактна. Так как $[\mathfrak{m}, \mathfrak{m}] \subset \mathfrak{k}$, то (вешественное) подпространство $\mathfrak{g}^{*}=\mathfrak{k} \oplus \mathfrak{m}^{*}, \mathfrak{m}^{*}=i \mathfrak{m}$, комплексификации $\mathfrak{g}^{\mathbb{C}}=\mathfrak{g} \oplus i \mathfrak{g}$ является (некомпактной) алгеброй Ли. Пусть $G^{*}-$ связная группа Ли с алгеброй Ли $\mathfrak{g}^{*}$ такая, что $G^{*} / K$ - симметрическое пространство. Тогда отображения $P: w \mapsto P_{w} \subset \mathrm{GL}\left(\mathfrak{m}^{\mathbb{C}}\right), W \subset \mathfrak{m}$, и $P^{(*)}: i w \rightarrow P_{w}$ удовлетворяют условию (3.1) одновременно.

4.1. Инвариантные кэлеровы структуры на инвариантных областях $D$ в касательных расслоениях пространств $\mathrm{SO}(n+1) / \mathrm{SO}(n)$ и $\mathrm{SO}_{0}(1, n) / \mathrm{SO}(n)(n \geqslant 2)$. Пусть $G$ - группа Ли $\mathrm{SO}(n+1)$ и $K$ - ее подгруппа, изоморфная $\mathrm{SO}(n)$. Пусть $\langle\langle\cdot, \cdot\rangle\rangle$ обозначает нормализованную форму $-\frac{\varepsilon}{2} \operatorname{Tr}$ на $\mathfrak{g}=\operatorname{so}(n+1) \mathrm{c} \varepsilon=1$, ассоциированную со стандартным представлением алгебры $\mathrm{so}(n+1)$. Для симметрического пространства $G / K=\mathrm{SO}(n+1) / \mathrm{SO}(n)$ его тензор кривизны задается формулой (см. [14; гл. Х, § 2])

$$
[w,[\xi, \eta]]=\varepsilon(\langle\langle w, \eta\rangle \xi-\langle\langle w, \xi\rangle\rangle \eta), \quad \varepsilon=1, \quad \forall w, \xi, \eta \in \mathfrak{m} .
$$

Мы попытаемся описать все отображения $P_{w}: W \rightarrow S\left(\mathfrak{m}^{\mathbb{C}}\right)$, для которых выполнено условие (3.1), такие, что каждый оператор $P_{w}$ имеет положительно определенную вешественную часть. Предположим, что $n \geqslant 3$. Так как Ad-представление $R_{\mathfrak{m}}$ группы Ли $K=\mathrm{SO}(n)$ в пространстве $\mathfrak{m}$ - это стандартное неприводимое $n$-мерное представление группы $\mathrm{SO}(n)$, то группа $K$ действует транзитивно на $(n-1)$-мерной сфере, состояшей из векторов пространства $\mathfrak{m}$ постоянной длины, и стационарная подгруппа $K_{w}$ изоморфна $\mathrm{SO}(n-1)$ для произвольного ненулевого $w \in W$. Следовательно, $\mathfrak{m}=\langle w\rangle \oplus\langle w\rangle^{\perp}$, где $\langle w\rangle$ - одномерньй тривиальный $K_{w}$-модуль, порожденный вектором $w$, и $\langle w\rangle^{\perp}-$ простой $K_{w}$-модуль, состоящий из всех векторов, ортогональных к $w$. В силу условия (4.1) для произвольного вектора $\xi \in \mathfrak{m}$ его две компоненты, соответствующие разложению $\mathfrak{m}=\langle w\rangle \oplus\langle w\rangle^{\perp}$, являются собственньпи векторами оператора $P_{w}$ с собственными значениями $\lambda(w)$ и $\psi(w)$ соответственно $(n \geqslant 3)$. Так как скалярное произведение $\langle\langle\cdot, \cdot\rangle\rangle \operatorname{Ad} K$-инвариантно, то из (3.1) следует, что $\lambda$ и $\psi$ - $\mathrm{Ad} K$-инвариантные комплекснозначные функции, т.е. $\lambda=\lambda(\|w\|), \psi=\psi(\|w\|)$, и

$$
P_{w}=\psi(\mathbf{r}) \cdot \operatorname{Id}+(\lambda(\mathbf{r})-\psi(\mathbf{r})) \mathbf{r}^{-2} \cdot w \otimes w, \quad w \in W^{0} .
$$

Если $n=2$ и $P_{w}(w)=\lambda(w) w$, то отображение $P$ также имеет вид $(4.3)(\operatorname{dim} \mathfrak{m}=2)$.

Положим $\Lambda(\mathbf{r})=(\lambda(\mathbf{r})-\psi(\mathbf{r})) \mathbf{r}^{-2}$. Как следует из предложения 3.3, отображение (4.3) определяет инволютивное распределение тогда и только тогда, когда $[\overrightarrow{P(\xi)}, \overrightarrow{P(\eta)}](w)=-[w,[\xi, \eta]]$. Нетрудно проверить, что

$$
\begin{aligned}
& {\left[\psi(\mathbf{r}) \xi_{b} \frac{\partial}{\partial w_{b}}+\Lambda(\mathbf{r})\langle\langle w, \xi\rangle\rangle w_{b} \frac{\partial}{\partial w_{b}}, \psi(\mathbf{r}) \eta_{b} \frac{\partial}{\partial w_{b}}+\Lambda(\mathbf{r})\left\langle\langle w, \eta\rangle w_{b} \frac{\partial}{\partial w_{b}}\right]\right.} \\
& \quad=\left(\mathbf{r}^{-1} \psi(\mathbf{r}) \psi^{\prime}(\mathbf{r})+\mathbf{r} \Lambda(\mathbf{r}) \psi^{\prime}(\mathbf{r})-\Lambda(\mathbf{r}) \psi(\mathbf{r})\right) \cdot\left(\langle\langle w, \xi\rangle\rangle \eta-\langle\langle w, \eta\rangle \xi)_{b} \frac{\partial}{\partial w_{b}} .\right.
\end{aligned}
$$

Учитывая (4.2) и заменяя $\Lambda(\mathbf{r})$ на $(\lambda(\mathbf{r})-\psi(\mathbf{r})) \mathbf{r}^{-2}$, мы получаем уравнение Риккати

$$
\psi^{\prime}(\mathbf{r})=-\frac{1}{\mathbf{r} \lambda(\mathbf{r})} \cdot \psi^{2}(\mathbf{r})+\frac{1}{\mathbf{r}} \psi(\mathbf{r})+\frac{\varepsilon \mathbf{r}}{\lambda(\mathbf{r})} .
$$


Используя его частное решение $\psi(\mathbf{r})=\mathbf{r}$, находим обшее решение:

$$
\psi(\mathbf{r})=\mathbf{r} \frac{C_{1} \exp (\alpha(\mathbf{r}))+C_{2} \exp (-\alpha(\mathbf{r}))}{C_{1} \exp (\alpha(\mathbf{r}))-C_{2} \exp (-\alpha(\mathbf{r}))}, \quad \alpha^{\prime}(\mathbf{r})=\frac{1}{\lambda(\mathbf{r})},
$$

где по крайней мере одна из двух комплексных констант $C_{1}, C_{2}$ ненулевая. Мы доказали следующую теорему.

Tеорема 4.3. Пусть $G=\operatorname{SO}(n+1)$ и $K$ - подгруппа в $G$, изоморфная $\mathrm{SO}(n)$. Пусть $F \in \mathfrak{K}(D)-G$-инвариантная кәлерова структура в области $D \subset T(G / K)$. Если $n \geqslant 3$, то $F=F(P)$, где отображсение $P: W \rightarrow S\left(\mathfrak{m}^{\mathbb{C}}\right)$ имеет вид (4.3). Если $n \geqslant 2$, то отображсение $P$ (4.3) определяет кәлерову структуру на $D^{0}$ тогда и только тогда, когда вещественные части гладких функиий $\lambda(\|w\|), \psi(\|w\|): W^{0} \rightarrow \mathbb{C}$ положительны и $\psi(\mathbf{r})$ - решение уравнения Риккати (4.4) с $\varepsilon=1$, т.е. $\psi(\mathbf{r})$ задается формулой (4.5).

ЗАмечание 4.4 . Так как форма $\langle\langle\cdot, \cdot\rangle$ Ad $G$-инвариантна, то вся конструкция целиком и доказательство теоремы 4.3 зависят существенно только от пары $(\mathfrak{g}, \mathfrak{k})=$ $(\operatorname{so}(n+1), \operatorname{so}(n))$ алгебр Ли. Учитьвая изоморфизмы [14; гл. $\mathrm{X}, \S 6.4]$

$$
\begin{gathered}
(\mathrm{su}(2), u(1)) \simeq(\operatorname{so}(3), \operatorname{so}(2)), \quad(\operatorname{sp}(2), \operatorname{sp}(1) \oplus \operatorname{sp}(1)) \simeq(\operatorname{so}(5), \operatorname{so}(4)), \\
(\operatorname{so}(3) \oplus \operatorname{so}(3), \operatorname{diag}(\operatorname{so}(3) \oplus \operatorname{so}(3))) \simeq(\operatorname{so}(4), \operatorname{so}(3)),
\end{gathered}
$$

мы получаем, что теорема справедлива также и для следующих симметрических пространств ранга один:

$$
\begin{gathered}
\mathbb{C} P^{1}=\mathrm{SU}(2) / S(U(1) \times U(1)), \quad \mathbb{H} P^{1}=\mathrm{Sp}(2) /(\mathrm{Sp}(1) \times \mathrm{Sp}(1)), \\
(\mathrm{SO}(3) \times \mathrm{SO}(3)) / \operatorname{diag}(\mathrm{SO}(3) \times \mathrm{SO}(3)) .
\end{gathered}
$$

Мы будем говорить, что симметрическое пространство $G / K$ - пространство $с ф е-$ рического типа, если $(\mathfrak{g}, \mathfrak{k}) \simeq(\mathrm{so}(n+1), \mathrm{so}(n)), n \geqslant 2$. Так как для таких пространств $\sqrt{-\mathrm{ad}^{2} w} \mid \mathfrak{m}=\|w\| \cdot \mathrm{Id}-\|w\|^{-1} \cdot w \otimes w($ см. (4.2)), то формула (3.10) получается как непосредственное следствие этой теоремы. Больше того, $F^{c}=F(P)$ с $P$ из (4.3), где $\lambda(\mathbf{r})=1$ и $\psi(\mathbf{r})=\mathbf{r} \operatorname{ch}(\mathbf{r}) / \operatorname{sh}(\mathbf{r})$.

Теперь пусть $G$ - связная группа Ли $\mathrm{SO}_{0}(1, n)$ и $K$ - ее подгруппа, изоморфная $\mathrm{SO}(n)$. Пусть $\left\langle\langle\cdot, \cdot\rangle\right.$ обозначает нормализованную форму $-\frac{\varepsilon}{2} \operatorname{Tr} \mathrm{c} \varepsilon=-1$ (на $\mathfrak{g}=$ so $(1, n))$, ассоциированную со стандартным представлением алгебры Ли $\mathrm{so}(1, n)$. Тогда для симметрического пространства $G / K=\mathrm{SO}_{0}(1, n) / \mathrm{SO}(n)$ имеет место формула (4.2) с $\varepsilon=-1$. Замечание 4.2 и анализ, подобный приведенному выше при доказательстве теоремы 4.3 , показывает, что

(1) если $n \geqslant 3$, то для отображения $P: W \rightarrow S\left(\mathfrak{m}^{\mathbb{C}}\right), w \mapsto P_{w}$, выполнено условие (3.1) тогда и только тогда, когда операторы $P_{w}$ имеют форму (4.3);

(2) распределение $F(P)$ инволютивно тогда и только тогда, когда $\psi$ является решением уравнения (4.4) с $\varepsilon=-1$.

В этом случае общее решение уравнения (4.4) задается формулой

$$
\psi(\mathbf{r})=i \mathbf{r} \frac{C_{1} \exp (i \alpha(\mathbf{r}))+C_{2} \exp (-i \alpha(\mathbf{r}))}{C_{1} \exp (i \alpha(\mathbf{r}))-C_{2} \exp (-i \alpha(\mathbf{r}))}, \quad \alpha^{\prime}(\mathbf{r})=\frac{1}{\lambda(\mathbf{r})},
$$

где по крайней мере одна из двух комплексных констант $C_{1}, C_{2}$ отлична от нуля. Таким образом, доказана 
Теорема 4.5. Пусть $G=\mathrm{SO}_{0}(1, n)$ и $K$ - подгруппа в $G$, изоморфная $\mathrm{SO}(n)$. Пусть $F \in \mathfrak{K}(D)-G$-инвариантная кэлерова структура в области $D \subset T(G / K)$. Если $n \geqslant 3$, то $F=F(P)$, где отображсение $P: W \rightarrow \mathrm{GL}\left(\mathfrak{m}^{\mathbb{C}}\right)$ имеет вид (4.3). Если $n \geqslant 2$, то отображсение $P$ (4.3) определяет кэлерову структуру на $D^{0}$ тогда и только тогда, когда вещественные части гладких функиий $\lambda(\|w\|), \psi(\|w\|): W^{0} \rightarrow \mathbb{C}$ положительны и $\psi(\mathbf{r})$ - решение уравнения Риккати (4.4) с $\varepsilon=-1$, т.е. $\psi(\mathbf{r})$ задается формулой (4.6).

4.2. $K$-эквивариантные отображения. В этом пункте мы опишем $K$-эквивариантные отображения $P: W^{0} \rightarrow S\left(\mathfrak{m}^{\mathbb{C}}\right)$ (т.е. удовлетворяющие условию $(3.1)$ ) для компактных симметрических пространств ранга один несферического типа.

А. Пусть $G$ - группа Ли $U(n+1), n \geqslant 2$, и $K$-ее подгруппа, изоморфная $U(1) \times$ $U(n)$. Пусть $\left\langle\langle\cdot, \cdot\rangle\right.$ обозначает нормализованную форму $-\frac{\varepsilon}{2} \operatorname{Tr}($ на $\mathfrak{g}=u(n+1))$ с $\varepsilon=1$, ассоциированную со стандартньм представлением алгебры Ли $u(n+1)$. Для симметрического пространства $G / K=U(n+1) /(U(1) \times U(n))$ его тензор кривизны задается формулой (см. [14; гл. Х, § 2])

$$
[w,[\xi, \eta]]=\varepsilon(\langle\langle w, \eta\rangle\rangle \xi+\langle\langle w, J \eta\rangle\rangle \xi-\langle\langle w, \xi\rangle\rangle \eta-\langle\langle w, J \xi\rangle\rangle J \eta+2\langle\langle\xi, J \eta\rangle J w),
$$

где $\varepsilon=1, w, \xi, \eta \in \mathfrak{m}, J: \mathfrak{m} \rightarrow \mathfrak{m}-\mathbb{R}$-линейный оператор, определяющий комплексную структуру на $\mathfrak{m}$. В частности, $J^{2}=-1$. Более того, эндоморфизм $J$ ортогонален относительно формы $\langle\cdot, \cdot\rangle$, т.е. $J^{*}=-J$. Так как Ad-представление подгруппы $K^{n} \subset K, K^{n}=U(n)$, в $\mathfrak{m}$ - это стандартное неприводимое $n$-мерное представление $U(n)$, то группа $K^{n}$ действует транзитивно на $(2 n-1)$-мерной сфере, состояшей из векторов пространства $\mathfrak{m}$ постоянной длины; стационарная подгруппа $K_{w}=K_{w}^{1} \times K_{w}^{n-1}$ изоморфна $U(1) \times U(n-1)$ для произвольного ненулевого $w \in W\left[14 ;\right.$ гл. Х]. Следовательно, $\mathfrak{m}=\langle w, J w\rangle \oplus\langle w, J w\rangle^{\perp}$, где $\langle w, J w\rangle$ - двумерный тривиальный $K_{w}^{n-1}$-модуль, порожденный векторами $w, J w$, и $\langle w, J w\rangle^{\perp}-$ простой $K_{w}^{n-1}$-модуль, состоящий из векторов, ортогональных к $w, J w$. Подгруппа $K_{w}^{1}$ группы $K_{w}$ действует тривиально на $\langle w, J w\rangle$ и как умножение на характер на $\langle w, J w\rangle^{\perp}$. Учитывая, что оператор $J$ коммутирует с Ad-действием группы $K$ на m, мы получаем, что произвольное $K$-эквивариантное отображение $P: W \rightarrow S\left(\mathfrak{m}^{\mathbb{C}}\right)$ задается формулой

$$
P_{w}=\psi(\mathbf{r}) \cdot \operatorname{Id}+a(\mathbf{r}) J w \otimes J w+b(\mathbf{r}) w \otimes J w+b(\mathbf{r}) J w \otimes w+A(\mathbf{r}) w \otimes w, \quad w \in W^{0},
$$

где $\psi, a, b, A$-гладкие функции и по определению $\xi \otimes \eta(w) \stackrel{\text { def }}{=} \xi\langle\langle\eta, w\rangle$.

Предположим, что $P(4.8)$ определяет кэлерову структуру на $D$. Рассмотрим коммутатор $Y=[\overrightarrow{P(\xi)}, \overrightarrow{P(\eta)}]$ двух векторных полей на $W^{0}$. Так как $\overrightarrow{P(\xi)}$ задается формулой

$$
\left(\psi(\mathbf{r}) \xi+a(\mathbf{r})\left\langle\langle J w, \xi\rangle J w+b(\mathbf{r})\left\langle\langle J w, \xi\rangle w+b(\mathbf{r})\langle\langle w, \xi\rangle J w+A(\mathbf{r})\langle\langle w, \xi\rangle\rangle w)_{b} \frac{\partial}{\partial w_{b}}\right.\right.\right.
$$

и $d \mathbf{r}(w)(\xi)=\mathbf{r}^{-1}(w)\langle\langle w, \xi\rangle$, то мы получим, что значение векторного поля $Y$ в точке $w$ является элементом векторного пространства, порожденного шестью векторами $\xi, J \xi, \eta, J \eta, w, J w$. Если $n \geqslant 3$, мы можем предположить, что эти векторы линейно независимы. Приравнивая коэффициенты при $J \eta$ в $Y(w)$ и - $[w,[\xi, \eta]](4.7)$ 
(используя несложные вычисления), мы получаем уравнение $-\psi(\mathbf{r})(b(\mathbf{r})\langle\langle w, \xi\rangle\rangle+$ $a(\mathbf{r})\langle\langle J w, \xi\rangle)=\varepsilon\langle\langle w, J \xi\rangle$, которое выполняется для произвольных $w, \xi, \eta$ в общем положении, если распределение $F(P)$ инволютивно. Следовательно, $b(\mathbf{r}) \equiv 0$ и $\psi(\mathbf{r}) a(\mathbf{r}) \equiv \varepsilon$. Если $n=2$, то чтобы получить последние два тождества, нужно рассмотреть соответствующие коэффициенты при $J \eta$ в точках $w=\xi, J \xi$. Другими словами, векторы $w$ и $J w$ - собственные векторы оператора $P_{w}$. Соответствующие собственные значения мы обозначим через $\lambda(\|w\|)$ и $\varphi(\|w\|)$. Таким образом, доказана

Лемма 4.6. Если $F(P)$ - кәлерова структура на области $D$ в $T(U(n+1) /$ $(U(1) \times U(n))), n \geqslant 2$, то отображение $P$ имеет форму

$$
P_{w}=\psi(\mathbf{r}) \cdot \operatorname{Id}+(\varphi(\mathbf{r})-\psi(\mathbf{r})) \mathbf{r}^{-2} \cdot J w \otimes J w+(\lambda(\mathbf{r})-\psi(\mathbf{r})) \mathbf{r}^{-2} \cdot w \otimes w
$$

где $w \in W^{0}$. Больие того, $\psi(\mathbf{r})(\varphi(\mathbf{r})-\psi(\mathbf{r})) \mathbf{r}^{-2} \equiv \varepsilon$.

В. Пусть $G$ - группа Ли $\operatorname{Sp}(n+1), n \geqslant 2$, и $K=K^{1} \times K^{n}$ - ее подгруппа, изоморфная $\mathrm{Sp}(1) \times \mathrm{Sp}(n)$. Пусть $\langle\cdot \cdot, \cdot\rangle$ обозначает нормализованную форму $-\frac{\varepsilon}{2} \operatorname{Tr}$ $($ на $\mathfrak{g}=\operatorname{sp}(n+1)) \mathrm{c} \varepsilon=1$, ассоциированную со стандартным представлением алгебры Ли $\mathrm{sp}(n+1)$ кватернионными матрицами. Для произвольного ненулевого $w \in W$ стационарная подгруппа $K_{w}=K_{w}^{1} \times K_{w}^{n-1}$ изоморфна $\operatorname{Sp}(1) \times \operatorname{Sp}(n-1)[14]$. Чтобы описать Ad-представление группы $K_{w}$ в $\mathfrak{m}$, рассмотрим группу Ли $\operatorname{Sp}(m)$ как кватернионную унитарную группу.

Пусть $\mathrm{GL}_{m}(\mathbb{H})$ обозначает группу всех обратимых кватернионных $(m \times m)$-матриц. Тогда $\operatorname{Sp}(m)=\left\{X \in \mathrm{GL}_{m}(\mathbb{H}): \bar{X}^{t} X=1_{m}\right\}$ и $\operatorname{sp}(m)=\left\{X \in \operatorname{Mat}_{m}(\mathbb{H})\right.$ : $\left.\bar{X}^{t}+X=0\right\}$. При этом отождествлении $4 n$-мерное вешественное пространство $\mathfrak{m}$ - это $n$-мерное правое векторное пространство $\mathbb{H}^{n},\left\langle\langle w, w\rangle=\sum_{j=1}^{n} q_{j} \bar{q}_{j}\right.$ $\left(w=\left(q_{1}, \ldots, q_{n}\right)\right)$ и Аd-действие групшы $K=K^{1} \times K^{n}$ на $\mathbb{H}^{n}$ имеет вид $(a, A)(w)=$ $A \cdot w \cdot a^{-1}$. Поэтому $\mathbb{H}^{n}$ - прямая сумма тривиального четырехмерного (над $\mathbb{R}$ ) $K_{w}^{n-1}$-модуля $\langle w \mathbb{H}\rangle=\{w \cdot q, q \in \mathbb{H}\}$ и простого $K_{w}^{n-1}$-модуля $\langle w \mathbb{H}\rangle^{\perp}$, состоящего из всех векторов из $\mathfrak{m}$, ортогональных каждому $w \cdot q, q \in \mathbb{H}$. Простая компонента $K_{w}^{1}=\operatorname{Sp}(1)$ действует на $\langle w \mathbb{H}\rangle$ сопряжением $w \cdot q \mapsto w \cdot a q a^{-1}\left(\operatorname{Ad} K_{w}(w)=w\right)$. Поэтому как вещественный $K_{w}$-модуль $\langle w \mathbb{H}\rangle$ равен $\langle w\rangle \oplus\left\langle E_{1} w, E_{2} w, E_{3} w\right\rangle$, где линейные операторы $E_{1}, E_{2}, E_{3}$ определяют кватернионную структуру на $\mathfrak{m}=\mathbb{H}^{n}$ :

$$
\begin{gathered}
E_{p}^{2}=-\mathrm{Id}, \quad E_{p}^{*}=-E_{p}, \quad p=1,2,3 \\
E_{1} E_{2}=-E_{2} E_{1}=E_{3}, \quad E_{2} E_{3}=-E_{3} E_{2}=E_{1}, \quad E_{3} E_{1}=-E_{1} E_{3}=E_{2} .
\end{gathered}
$$

Так как внутренний автоморфизм $q \mapsto a q a^{-1}$ тела кватернионов $\mathbb{H}$ оставляет инвариантным подпространство $\mathbb{H}^{\prime}$ чисто мнимых кватернионов, то подпространство $\operatorname{Ad} k\left\langle E_{p} w, p=1,2,3\right\rangle$ совпадает с $\left\langle E_{p}\left(\operatorname{Ad}_{k} w\right), p=1,2,3\right\rangle\left(A \cdot w q^{\prime} \cdot a^{-1}=\right.$ $\left.\left(A \cdot w \cdot a^{-1}\right)\left(a q^{\prime} a^{-1}\right)\right)$. Используя представление групшы Ли $\operatorname{Sp}(n+1)$ кватернионньми матрицами, мы выведем формулу для коммутатора $[w,[\xi, \eta]], w, \xi, \eta \in \mathfrak{m}$. В терминах операторов $E_{p}, p=1,2,3$, и $E_{0}=\operatorname{Id}$ эта формула может быть записана в виде

$$
[w,[\xi, \eta]]=\varepsilon\left(\sum_{j=0}^{3}\left\langle\left\langle w, E_{j} \eta\right\rangle\right\rangle E_{j} \xi-\sum_{j=0}^{3}\left\langle\left\langle w, E_{j} \xi\right\rangle\right\rangle E_{j} \eta+2 \sum_{p=1}^{3}\left\langle\left\langle\xi, E_{p} \eta\right\rangle\right\rangle E_{p} w\right)
$$


где $\varepsilon=1$. В силу условия (3.1) для произвольного вектора $\xi \in \mathfrak{m}$ его три компоненты, соответствующие разложению $\mathfrak{m}=\langle w\rangle \oplus\left\langle E_{1} w, E_{2} w, E_{3} w\right\rangle \oplus\langle w \mathbb{H}\rangle^{\perp}$, являются собственными векторами оператора $P_{w}$ с собственньми значениями $\lambda(w)$, $\varphi(w)$ и $\psi(w)$ соответственно. Из (4.9) вытекает, что отображение $P: W \rightarrow S\left(\mathfrak{m}^{\mathbb{C}}\right)$ $K$-эквивариантно тогда и только тогда, когда для всех $w \in W^{0}$

$$
P_{w}=\psi(\mathbf{r}) \cdot \operatorname{Id}+(\varphi(\mathbf{r})-\psi(\mathbf{r})) \mathbf{r}^{-2} \cdot \sum_{p=1}^{3} E_{p} w \otimes E_{p} w+(\lambda(\mathbf{r})-\psi(\mathbf{r})) \mathbf{r}^{-2} \cdot w \otimes w
$$

Лемма 4.7. Если $F(P)$ - кәлерова структура на области $D$ в $T(\operatorname{Sp}(n+1) /$ $(\operatorname{Sp}(1) \times \operatorname{Sp}(n))), n \geqslant 2$, то $P$ имеет вид $(4.11)$, где $\psi(\mathbf{r})(\varphi(\mathbf{r})-\psi(\mathbf{r})) \mathbf{r}^{-2} \equiv \varepsilon$.

ДоказАтельство. Рассмотрим коммутатор $Y=[\overrightarrow{P(\xi)}, \overrightarrow{P(\eta)}]$ двух векторных полей на $W^{0}$. Из формулы (4.11) вытекает, что значение векторного поля $Y$ в точке $w$ является элементом векторного пространства, порожденного двенадцатью векторами: $\xi, \eta, w$ и их образами при $E_{j}, j=1,2,3$ (восемью, если $w=E_{1} \xi$ ). Приравнивая коэффициенты при $E_{1} \eta$ в $Y(w)$ и $-[w,[\xi, \eta]](4.10)$ в точке $w=E_{1} \xi$ (с помощью несложных вычислений), мы получаем условие леммы при $n \geqslant 2$.

C. В этом пункте мы рассмотрим симметрическое пространство $G / K=$ $F_{4} / \operatorname{Spin}(9)$, единственное особое компактное симметрическое пространство ранга один. Пусть $(\langle\cdot, \cdot\rangle=c \Phi) \mid \mathfrak{m}$ - положительно определенное скалярное произведение на $\mathfrak{m}$. Представление $R_{\mathfrak{m}}$ групшы Ли $K=\operatorname{Spin}(9)$ в $\mathfrak{m}-$ это точное вешественное представление в 16-мерном пространстве [14], [15], единственное неприводимое представление группы Ли $\operatorname{Spin}(9)$ размерности 16 [16]. Более того, $K=\operatorname{Spin}(9)$ действует транзитивно на 15-мерной сффере, состояшей из всех векторов из $\mathfrak{m}$ постоянной длины; стационарная подгруппа $K_{w}$ произвольного ненулевого элемента $w \in \mathfrak{m}$ изоморфна $\operatorname{Spin}(7)$ [16; гл. I, §5, пример 5]. Но для $F_{4} / \operatorname{Spin}(9)$ система положительных ограниченных корней $\Sigma^{+}$содержит только два элемента $\sigma$ и $2 \sigma$ с кратностями $m_{\sigma}=8$ и $m_{2 \sigma}=7$ [14; таблица VI]. Поэтому в силу замечания $4.1 \quad K_{w}$-модуль $\mathfrak{m}$ есть прямая сумма $\langle w\rangle \oplus V_{7}(w) \oplus V_{8}(w)$ одномерного тривиального $K_{w}$-модуля $\langle w\rangle$ и двух $K_{w}$-модулей $V_{7}(w)$ и $V_{8}(w)$ размерностей 7 и 8 . Ясно, что $V_{7}(w)$ и $V_{8}(w)$ - простые $K_{w}$-модули (см. [16; гл. I, $\S 5$, примеры 4,5$])$. Чтобы описать $K_{w}$-модуль $V_{8}(w)$, мы рассмотрим конструкцию спинорного представления группы Ли $\operatorname{Spin}(9)$ более детально.

Пусть $V$ - вешественное пространство размерности 9 , снабженное положительно определенной билинейной формой $Q$. Пусть $e_{0}, \ldots, e_{8}-$ ортонормированньй базис в $V$. В терминах этого базиса алгебра Клиффорда $\mathbb{C} l_{+}(9)$ определяется как вешественная ассоциативная алгебра с единицей 1 , генераторами $e_{0}, \ldots, e_{8}$ и определяюшими соотношениями

$$
e_{j} \cdot e_{k}+e_{k} \cdot e_{j}=0, \quad j \neq k, \quad e_{j}^{2}=1, \quad j, k=0, \ldots, 8 .
$$

Пусть $\operatorname{pin}_{+}(9)$ - мультипликативная подгруппа групшы всех обратимых элементов из $\mathbb{C} l_{+}(9)$, порожденная векторами длины один из $V$. Если $Q(v, v)=1$, то $v \cdot v=1$, т.е. $v \in \operatorname{pin}_{+}(9)$. Группа Ли $\operatorname{Spin}_{+}(9) \simeq \operatorname{Spin}(9)$ - это подгрупша группы $\operatorname{pin}_{+}(9)$, состоящая из четных элементов, т.е.

$$
\operatorname{Spin}(9)=\left\{v_{1} \cdot v_{2} \cdots v_{2 k}, Q\left(v_{j}, v_{j}\right)=1, j=1, \ldots, 2 k, k \in \mathbb{N}\right\} .
$$


Более того, группа $\operatorname{Spin}(9)$ сохраняет при сопряжении пространство $V: g V g^{-1}$ $=V[17 ;$ лекция 13$]$.

Сушествует точное 16-мерное представление группы $\operatorname{pin}_{+}(9)$ ортогональными матрицами [17; лекция 15]. Другими словами, $\operatorname{Spin}(9) \subset \operatorname{pin}_{+}(9) \subset O(16)$. Поэтому найдутся девять ортогональных (относительно формы $\langle\cdot, \cdot\rangle$ на $\mathfrak{m}$ ) линейных преобразований $E_{k}: \mathfrak{m} \rightarrow \mathfrak{m}$, удовлетворяюших соотношениям

$$
E_{j} E_{k}+E_{k} E_{j}=0, j \neq k, \quad E_{j}^{2}=\mathrm{Id}, E_{j}^{*}=E_{j}^{-1}=E_{j}, \quad j, k=0, \ldots, 8 .
$$

В силу соотношений (4.13) для произвольного ненулевого $w \in \mathfrak{m}$ векторы $E_{j} w, j=$ $0, \ldots, 8$, образуют ортогональный базис некоторого подпространства $V_{9}(w) \subset \mathfrak{m}$. Более того, так как $g V g^{-1}=V$ для каждого $g \in \operatorname{Spin}(9)$, то каждый эндоморфизм $g E_{j} g^{-1} \in \operatorname{End}(\mathfrak{m})$ является линейной комбинацией эндоморфизмов $E_{j}$, $j=0, \ldots, 8$. Следовательно, если $g \in \operatorname{Ad} K_{w}$, то $g\left(V_{9}(w)\right)=V_{9}(w)$. Из соображений размерности получим разложение $V_{9}(w)=\langle w\rangle \oplus V_{8}(w)$.

Теперь пусть $P: W^{0} \rightarrow S\left(\mathfrak{m}^{\mathbb{C}}\right)$ - отображение, для которого выполнено условие (4.1). Тогда пространства $\langle w\rangle, V_{8}(w)$ и $V_{7}(w)$ являются собственньпи подпространствами оператора $P_{w}$ с собственными значениями $\lambda(w), \varphi(w)$ и $\psi(w)$ соответственно. Как следует из изложенного выше и соотношения (3.1), отображение $P: W^{0} \rightarrow S\left(\mathfrak{m}^{\mathbb{C}}\right) K$-эквивариантно тогда и только тогда, когда для всех $w \in W^{0}$

$$
P_{w}=\psi(r) \cdot \operatorname{Id}+(\varphi(r)-\psi(r)) r^{-2} \cdot \sum_{j=0}^{8} E_{j} w \otimes E_{j} w+(\lambda(r)-\varphi(r)) r^{-2} \cdot w \otimes w
$$

4.3. Необходимое условие. Пусть $G / K$ - симметрическое пространство ранга один (компактного или некомпактного типа). Пусть $(\langle\cdot, \cdot\rangle=c \Phi) \mid \mathfrak{m}-$ положительно определенное скалярное произведение на $\mathfrak{m}$ и $r(w)=|w|=\sqrt{\langle w, w\rangle}$. Следуюшее предложение подытоживает результаты, доказанные в предыдуших пунктах (если мы положим $E_{0}=\mathrm{Id}$ в классическом случае, $E_{1}=J$ для $\mathbb{C} P^{n}$ $(n \geqslant 2), \psi=\varphi$ для $G / K$ сферического типа и применим замечание 4.2 в некомпактном случае).

ПРЕДЛОЖЕНИЕ 4.8. Пусть $G / K$ - симметрическое пространство ранга один (со связной компактной подгруппой $K$ ). Тогда существует множество ортогональных эндоморфизмов $E_{j}: \mathfrak{m} \rightarrow \mathfrak{m}, j=0, \ldots, N, N=N(G / K) \in$ $\{0,1,3,8\}$, удовлетворяющих соотношениям $n .4 .2$ и таких, что

(1) для каждого $k \in K$ әндоморфизм $\mathrm{Ad}_{\mathfrak{m}} k E_{j} \operatorname{Ad}_{\mathfrak{m}} k^{-1}$ является линейной комбинацией $E_{j}: \mathfrak{m} \rightarrow \mathfrak{m}, j=0, \ldots, N$;

(2) произвольное $K$-эквивариантное отображение $P: W^{0} \rightarrow S\left(\mathfrak{m}^{\mathbb{C}}\right)$, $P_{w}(w)=\lambda(w) w$, задается формулой

$$
P_{w}=\psi(r) \cdot \operatorname{Id}+(\varphi(r)-\psi(r)) r^{-2} \cdot \sum_{j=0}^{N} E_{j} w \otimes E_{j} w+(\lambda(r)-\varphi(r)) r^{-2} \cdot w \otimes w
$$


Пусть $P(\psi, \varphi, \lambda)$ обозначает отображение, заданное формулой (4.14). Мы попытаемся описать некоторые определяюшие соотношения между функциями $\psi, \varphi, \lambda$ для кэлеровой структуры $F(P), P=P(\psi, \varphi, \lambda)$, на $D \subset T(G / K)$. Прежде всего, мы найдем формулу для эндоморфизма $\sqrt{-\mathrm{ad}^{2} w} \mid \mathfrak{m}$ в компактном случае.

Предположим, что $G / K$ имеет компактный тип. В силу предложения 3.10 $\sqrt{-\mathrm{ad}^{2} w} \mid \mathfrak{m}+\lambda(r) r^{-2} \cdot w \otimes w-K$-эквивариантное отображение для произвольной положительной функции $\lambda$, т.е. совпадает с отображением $P_{w}\left(\psi_{0}, \varphi_{0}, \lambda\right)$, где $\psi_{0}, \varphi_{0}$ - некоторые гладкие функции на интервале $(0, \infty)$, в частности $\sqrt{-\mathrm{ad}^{2} w} \mid \mathfrak{m}=$ $P_{w}\left(\psi_{0}, \varphi_{0}, 0\right)$. Это отображение $P_{w}\left(\psi_{0}, \varphi_{0}, \lambda\right)$ удовлетворяет условию (3.2) (см. предложение 3.6). Так как $\lambda(r)>0$ и три эндоморфизма Id, $\sum_{j=0}^{N} E_{j} w \otimes E_{j} w$, $w \otimes w$ линейно независимы (два эндоморфизма Id, $w \otimes w$, если $G / K$ - пространство сфферического типа) и по определению $P_{w}^{2}\left(\psi_{0}, \varphi_{0}, \lambda\right)=P_{w}\left(\psi_{0}^{2}, \varphi_{0}^{2}, \lambda^{2}\right)$, мы получим из $(3.2)$, что $\psi_{0}^{\prime}=r^{-1} \psi_{0}$ и $\varphi_{0}^{\prime}=r^{-1} \varphi_{0}$. Следовательно, $\psi_{0}(r)=c_{1} r$ и $\varphi_{0}(r)=c_{2} r$. Другими словами,

$$
\sqrt{-\mathrm{ad}^{2} w} \mid \mathfrak{m}=c_{1} r \cdot \operatorname{Id}+\left(c_{2}-c_{1}\right) r^{-1} \cdot \sum_{j=0}^{N} E_{j} w \otimes E_{j} w-c_{2} r^{-1} \cdot w \otimes w .
$$

Так как алгебра $\mathfrak{g}$ компактна, то константы $c_{1}$ и $c_{2}$ положительны. Ввиду замечания 4.1 и того, что было сказано в предыдущем пункте, $c_{2} / c_{1}=2$ для комплексного и кватернионного проективных пространств $(n \geqslant 2), c_{2} / c_{1}=1 / 2$ для проективной плоскости Кэли. Мы положим $c_{2} / c_{1}=1$ для симметрических пространств сферического типа $\left(S^{n}, \mathbb{C} P^{1}, \mathbb{H} P^{1},(\mathrm{SO}(3) \times \mathrm{SO}(3)) / \mathrm{DSO}(3)\right)$. Нетрудно заметить, учитывая сказанное (см. (4.2), (4.7), (4.10)), что в классическом случае существует единственное скалярное произведение $\left\langle\langle\cdot, \cdot\rangle=-\frac{1}{2} \operatorname{Tr}\right.$ на $\mathfrak{g}$, для которого $c_{1}=1$. Теперь следуюшее предложение очевидно.

ПРЕДЛОЖЕНИЕ 4.9. Пусть $G / K=F_{4} / \operatorname{Spin}(9)$ и әндоморфизмы $\left\{E_{j}, j=\right.$ $0, \ldots, 8\}$ удовлетворяют определяющим соотношениям (4.13). Существует единственное положительно определенное скалярное произведение $\langle\cdot, \cdot\rangle=$ $c \Phi$ на компактной алгебре Ли $\mathfrak{g}=\mathfrak{g}\left(F_{4}\right)$ такое, что эндоморфизм $\sqrt{-\mathrm{ad}^{2} w} \mid \mathfrak{m}$ задается формулой (4.15) с константой $c_{1}=2$. Тогда $c_{2}=1 u$

$$
-\operatorname{ad}^{2} w \mid \mathfrak{m}=4\left\langle\langle w, w\rangle \cdot \operatorname{Id}-3 \sum_{j=0}^{8} E_{j} w \otimes E_{j} w-w \otimes w .\right.
$$

ЗАмЕчАнИЕ 4.10. Если $G^{*} / K$ - дуальное симметрическое пространство некомпактного типа, то нормализованное скалярное произведение $\langle\langle\cdot, \cdot\rangle$ на компактной алгебре Ли $\mathfrak{g}$ определяет нормализованное скалярное произведение на $\mathfrak{m}^{*}=i \mathfrak{m}$ следуюшим образом: $\left(w^{*}, w^{*}\right)=(i w, i w) \mapsto\langle\langle w, w\rangle, w \in \mathfrak{m}$ (см. замечание 4.2).

Лемма 4.11. Если $F(P)$ - кәлерова структура на области $D$ в $T(G / K)$ и $P$ имеет вид (4.14), то функиии $\psi(\mathbf{r})$ и $\varphi(\mathbf{r})$ являются решениями уравнения Риккати

$$
y^{\prime}(\mathbf{r})=-\frac{1}{\mathbf{r} \lambda(\mathbf{r})} \cdot y^{2}(\mathbf{r})+\frac{1}{\mathbf{r}} y(\mathbf{r})+\frac{C \mathbf{r}}{\lambda(\mathbf{r})}
$$

с $C=\varepsilon c_{1}^{2}$ и $C=\varepsilon c_{2}^{2}$ соответственно, где $\varepsilon=1$, если пространство $G / K$ компактного типа, $и \varepsilon=-1$ в противном случае. 
ДоказАТельство. В силу предложения 3.6 отображение $P$ удовлетворяет условию (3.2). Предположим, что полупростая группа Ли $G$ компактна, т.е. $G / K-$ пространство компактного типа и $\varepsilon=1$. Учитьвая формулы (4.14) и (4.15), мы получаем, что левая часть уравнения (3.2) есть линейная комбинация трех эндоморфизмов Id, $\sum_{j=0}^{N} E_{j} w \otimes E_{j} w$ и $w \otimes w$. Нетрудно проверить, что соответствующие коэффициенты обращаются в нуль только в том случае, когда условия леммы вьполнены. Применяя замечания 4.2 и 4.10 , мы завершаем доказательство.

ЗАмЕчАниЕ 4.12. Непосредственная проверка показывает, что $\psi(\mathbf{r})$ есть всюду отличное от нуля на $O \subset[0, \infty)$ решение уравнения Риккати $(4.16)$ с $C=\varepsilon$ тогда и только тогда, когда функция $\varphi(\mathbf{r})=\psi(\mathbf{r})+\varepsilon \mathbf{r}^{2} / \psi(\mathbf{r})$ есть решение уравнения (4.16) с $C=4 \varepsilon$ (этот факт следует из лемм 4.6 и 4.7). Такие решения $\psi_{ \pm}$ имеют вид (4.5), (4.6), а $\varphi_{ \pm}$имеют следуюший вид

$$
\begin{aligned}
& \varphi_{+}(\mathbf{r})=2 \mathbf{r} \frac{C_{1}^{2} e^{2 \alpha(\mathbf{r})}+C_{2}^{2} e^{-2 \alpha(\mathbf{r})}}{C_{1}^{2} e^{2 \alpha(\mathbf{r})}-C_{2}^{2} e^{-2 \alpha(\mathbf{r})}}, \\
& \varphi_{-}(\mathbf{r})=2 i \mathbf{r} \frac{C_{1}^{2} e^{2 i \alpha(\mathbf{r})}+C_{2}^{2} e^{-2 i \alpha(\mathbf{r})}}{C_{1}^{2} e^{2 i \alpha(\mathbf{r})}-C_{2}^{2} e^{-2 i \alpha(\mathbf{r})}},
\end{aligned}
$$

где $\alpha^{\prime}(\mathbf{r})=1 / \lambda(\mathbf{r})$.

4.4. Основная лемма. Здесь мы докажем для проективной плоскости Кэли лемму, аналогичную леммам 4.6 и 4.7 . В этом пункте $G / K$ обозначает особое компактное симметрическое пространство $F_{4} / \operatorname{Spin}(9)$. Мы используем обозначения пунктов $4.2 \mathrm{C}$ и 4.3. Для произвольных векторов $w, \xi, \eta \in \mathfrak{m}$ мы будем писать $\xi \stackrel{V_{9}(w)}{=} \eta$, если $\xi-\eta \in V_{9}(w)$, где $V_{9}(w)$ - подпространство в $\mathfrak{m}$, порожденное векторами $E_{j} w, j=0, \ldots, 8$.

Предположим, что $K$-эквивариантное отображение $P(\psi, \varphi, \lambda)$ определяет кэлерову структуру на области $D \subset T(G / K)$. Рассмотрим коммутатор $Y(\xi, \eta)=$ $[\overrightarrow{P(\xi)}, \overrightarrow{P(\eta)}]$ двух векторных полей на $W^{0}=W^{0}(D)$. Используя (4.14), нетрудно показать, что

$$
\begin{aligned}
Y(\xi, \eta)(w) \stackrel{V_{9}(w)}{=} & \psi^{\prime}(\mathbf{r}) \mathbf{r}^{-1}\left(\left\langle\left\langle, P_{w}(\xi)\right\rangle\right\rangle-\left\langle\left\langle w, P_{w}(\eta)\right\rangle\right\rangle \xi\right) \\
& +(\lambda(\mathbf{r})-\varphi(\mathbf{r})) \psi(\mathbf{r}) \mathbf{r}^{-2}(\langle\langle, \eta\rangle\rangle \xi-\langle\langle w, \xi\rangle\rangle \eta) \\
& +(\varphi(\mathbf{r})-\psi(\mathbf{r})) \psi(\mathbf{r}) \mathbf{r}^{-2}\left(\sum_{j=0}^{8}\left\langle\left\langle E_{j} w, \eta\right\rangle\right\rangle E_{j} \xi-\sum_{j=0}^{8}\left\langle\left\langle E_{j} w, \xi\right\rangle E_{j} \eta\right)\right. \\
& +2(\varphi(\mathbf{r})-\psi(\mathbf{r}))^{2} \mathbf{r}^{-4} \sum_{\substack{j, k=0 \\
k \neq j}}^{8}\left\langle\left\langle E_{k} w, \eta\right\rangle\right\rangle\left\langle E_{j} w, \xi\right\rangle E_{k} E_{j} w .
\end{aligned}
$$

Учитьвая, что $\sqrt{-\mathrm{ad}^{2} w} \mid \mathfrak{m}=P_{w}(2 \mathbf{r}, \mathbf{r}, 0)$, из соотношений $(4.13)$ и следст- 
вия 3.10.1 вытекает, что для произвольных $w, \xi, \eta \in \mathfrak{m}$

$$
\begin{aligned}
-[w,[\xi, \eta]]= & 2\langle\langle w, \xi\rangle\rangle \eta-2\left\langle\langle w, \eta\rangle \xi+2 \sum_{j=0}^{8}\left\langle\left\langle E_{j} w, \xi\right\rangle\right\rangle E_{j} \eta\right. \\
& -2 \sum_{j=0}^{8}\left\langle\left\langle E_{j} w, \eta\right\rangle\right\rangle E_{j} \xi+2 \mathbf{r}^{-2} \sum_{\substack{j, k=0 \\
k \neq j}}^{8}\left\langle\left\langle E_{k} w, \eta\right\rangle\right\rangle\left\langle\left\langle E_{j} w, \xi\right\rangle\right\rangle E_{k} E_{j} w \\
& +\mathbf{r}^{-2} \sum_{\substack{j, k=0 \\
k \neq j}}^{8}\left(\left\langle\left\langle E_{k} E_{j} w, \eta\right\rangle\right\rangle\left\langle\left\langle E_{j} w, \xi\right\rangle\right\rangle-\left\langle\left\langle E_{k} E_{j} w, \xi\right\rangle\left\langle\left\langle E_{j} w, \eta\right\rangle\right\rangle\right) E_{k} w .\right.
\end{aligned}
$$

Лемма 4.13. Если $F(P)$ - кэлерова структура на области $D$ в $T\left(F_{4} /\right.$ $\operatorname{Spin}(9))$, то $P=P(\psi, \varphi, \lambda)$ имеет вид (4.14), где $\varphi(\mathbf{r})(\psi(\mathbf{r})-\varphi(\mathbf{r})) \mathbf{r}^{-2} \equiv \varepsilon$, $\varepsilon=1$.

ДокАЗАТЕльство. Так как $P(\psi, \varphi, \lambda)$ определяет кэлерову структуру на $D=D(W)$, тождество $Y(\xi, \eta)(w)=-[w,[\xi, \eta]]$ выполнено для каждого $\xi, \eta \in \mathfrak{m}$, $w \in W$ (см. предложение 3.3$)$. Вследствие ортогональности базиса $\left\{E_{p} w, p=\right.$ $0, \ldots, 8\}$ пространства $V_{9}(w)$ мы сушественно упростим вычисления, рассматривая значение векторного поля $Y\left(E_{p} w, E_{l} w\right), p \neq l$, в точке $w \neq 0$. Тогда из $(4.17)$, (4.18) и определяющих соотношений (4.13) вытекает, что $Y\left(E_{p} w, E_{l} w\right)(w) \stackrel{V_{9}(w)}{=}$ $-\left[w,\left[E_{p} w, E_{l} w\right]\right]$ тогда и только тогда, когда $\varphi(\mathbf{r})(\varphi(\mathbf{r})-\psi(\mathbf{r})) E_{l} E_{p} w \stackrel{V_{9}(w)}{=}$ $-\mathbf{r}^{2} E_{l} E_{p} w, l \neq p$. Другими словами, условие леммы выполнено, если $E_{l} E_{p} w \notin$ $V_{9}(w)$ для некоторых $p, l \in\{0, \ldots, 8\}$. В противном случае если $E_{l} E_{p} w \in V_{9}(w)$ для всех $0 \leqslant p, l \leqslant 8$, то каждый оператор $E_{p}$ оставляет инвариантным пространство $V_{9}(w)$ и, следовательно, в силу (4.12) подпространство $V_{9}(w)$ Ad $K$-инвариантно. Это противоречит простоте $\operatorname{Spin}(9)$-модуля $\mathfrak{m}$.

4.5. Инвариантные кэлеровы структуры на инвариантных областях $D$. Пусть $G / K$ - симметрическое пространство ранга один (компактного или некомпактного типа). Пусть 《,$\cdot \cdot\rangle$ обозначает нормализованное положительно определенное скалярное произведение на $\mathfrak{m}$ (см. замечание 4.10). Положим $\varepsilon=1$, если $G / K$ имеет компактный тип, и $\varepsilon=-1$ в противном случае. Напомним, что пространство $G / K$ сферического типа, если $(\mathfrak{g}, \mathfrak{k}) \simeq(\operatorname{so}(n+1), \operatorname{so}(n)), n \geqslant 2$.

Теорема 4.14. Пусть $G / K$ - симметрическое пространство ранга один c полупростой связной группой Ли $G$ и связной компактной подгруппой $K$. Пусть $F-G$-инвариантная кәлерова структура на области $D \subset T(G / K)$. Eсли $\operatorname{dim} G / K \geqslant 3$, mо $F=F(P)$, әде $K$-әквивариантное отобрахсение $P: W \rightarrow S\left(\mathfrak{m}^{\mathbb{C}}\right)$ имеет вид (4.14). Отображсене $P=P(\psi, \varphi, \lambda)$ (4.14) определяет кәлерову структуру на $D^{0}$ тогда и только тогда, когда

(1) вещественные части гладких функиий $\lambda(\|w\|), \psi(\|w\|), \varphi(\|w\|): W^{0} \rightarrow \mathbb{C}$ положительнь;

(2) $\psi(\mathbf{r})$ (соответственно $\varphi(\mathbf{r})$ ) есть решение уравнения Риккати (4.4), если $G / K$ - классическое симметрическое пространство (соответственно $G / K$ - особое симметрическое пространство); 
(3) $\psi(\mathbf{r})=\varphi(\mathbf{r})$, если пространство $G / K$ или дуальное к нему $G^{*} / K$ имеет сферический тип; $\psi(\mathbf{r})(\varphi(\mathbf{r})-\psi(\mathbf{r}))=\varepsilon \mathbf{r}^{2}$ (соответственно $\left.\varphi(\mathbf{r})(\psi(\mathbf{r})-\varphi(\mathbf{r}))=\varepsilon \mathbf{r}^{2}\right)$, если $G / K$ - иное классическое симметрическое пространство (соответственно $G / K$ - особое симметрическое пространство).

ДокАЗАТЕльство. Необходимость условий теоремы уже нами доказана (см. также замечание 4.4). Мы установим достаточность, используя следующую теорему Гийемина и Стензеля.

Теорема 4.15 [11]. Существуют $\sigma$-инвариантная окрестность $D^{c}$ нулевого сечения $G / K$ в $T(G / K)$ и единственная комплексная структура $F^{c}$ на $D^{c}$, удовлетворяющие двум условиям:

(1) б-антиголоморфная инволюиия;

(2) 1-форма $\operatorname{Im} \bar{\partial} H$ совпадает с канонической 1-формой $\theta$.

Более того, $F^{c}$ - кәлерова структура с кэлеровой формой $\Omega=i \bar{\partial} \partial H$.

Так как комплексная структура $g_{*}\left(F^{c}\right), g \in G$, также удовлетворяет условиям (1), (2) теоремы 4.15 , то $F^{c}-G$-инвариантная кэлерова структура. В силу леммы 3.1 и предложения $3.4 F^{c}=F\left(P^{c}\right)$, где $P_{w}^{c}(w)=w$, т.е. $\lambda(\mathbf{r}) \equiv 1$. Применяя теперь предложение 4.8 , мы получаем, что $P^{c}=P\left(\psi^{c}, \varphi^{c}, 1\right)$, где функции $\psi^{c}, \varphi^{c}$ - решения уравнения Риккати (4.16). Так как сушествует единственное решение уравнения (4.16) с $\lambda \equiv 1$, для которого соответствующая структура $F(P)$ регулярна в окрестности нулевого сечения $G / K \subset T(G / K)$, то мы получим, что

$$
\psi_{+}^{c}(\mathbf{r})=\frac{\mathbf{r} \operatorname{ch}(\mathbf{r})}{\operatorname{sh}(\mathbf{r})}, \varphi_{+}^{c}(\mathbf{r})=\psi_{+}^{c}(2 \mathbf{r}) \text { и } \quad \psi_{-}^{c}(\mathbf{r})=\frac{\mathbf{r} \cos (\mathbf{r})}{\sin (\mathbf{r})}, \varphi_{-}^{c}(\mathbf{r})=\psi_{-}^{c}(2 \mathbf{r})
$$

в классическом случае (если $G / K$ или дуальное к нему пространство $G^{*} / K$ имеет сферический тип, мы положим $\left.\varphi_{ \pm}^{c}=\psi_{ \pm}^{c}\right)$ и $\psi, \varphi$ нужно поменять местами в особом случае.

Пусть $F(P), P=P(\psi, \varphi, \lambda), \psi \neq \mathbf{r}(\psi \neq 2 \mathbf{r}$ в особом случае $),-$ распределение на $D$ со свойствами (1)-(3). Мы должны доказать только, что распределение $F(P)$ инволютивно. Предположим, что функции $\lambda$ и $\psi$ положительны. Тогда существует (локальная) положительная функция $\delta=\delta(\mathbf{r})$ такая, что $\lambda(\mathbf{r})=1 /(\mathbf{r} \delta(\mathbf{r}))^{\prime}$. Из (3.4), (4.5), (4.6) (см. также (3.9)) при подходящем выборе постоянной интегрирования вытекает, что локально $\Psi(\gamma)_{*}\left(F^{c}\right)=F(P)$, т.е. распределение $F(P)$ инволютивно.

Осталось доказать инволютивность распределения $F(P)$, когда $\lambda, \psi$-комплексные функции. Для этого зафиксируем векторы $\xi, \eta \in \mathfrak{m}$ и $w \in W^{0}$. Рассмотрим коммутатор $Y(w)=[\overrightarrow{P(\xi)}, \overrightarrow{P(\eta)}](w)$ как функцию от $\psi, \varphi, \lambda$ и их производных $\psi^{\prime}, \varphi^{\prime}, \lambda^{\prime}$. Так как оператор $P_{w}$ симметричен и $\psi, \varphi$ удовлетворяют уравнению Риккати (4.16), эта функция зависит только от переменных $\psi, \varphi, \lambda$. Более того, из условия (3) вытекает, что каждая компонента (координата) вектора $\lambda \psi^{2} Y(w)$ являет собой вещественный полином $f_{b}$ от переменных $\psi, \lambda$ степени $\leqslant 5$. Учитывая (4.5), (4.6) и то, что было сказано выше, получаем, что этот полином равен тождественно нулю на некотором открытом подмножестве в $\mathbb{R}^{2}$. Поэтому $f_{b} \equiv 0$. 


\section{$\S$ 5. Редукция}

5.1. Редукция и поляризации. В этом пункте мы изложим необходимые нам результаты работ [8], [18], модифицированные для наших нужд. Предположим, что $X$ - кокасательное расслоение $T^{*} N$ многообразия $N$. Пусть $p: T^{*} N \rightarrow N$ - каноническая проекция. Обозначим через $G$ и $S$ вещественные редуктивные связные группы Ли, которые действуют на $N$, и предположим, что эти действия коммутируют. Действия групп Ли $G, S$ на $N$ естественно продолжаются до действий $G, S$ на $T^{*} N$. Эти действия на $T^{*} N$ симплектические, так как они сохраняют каноническую 1-форму $\theta$, а значит, и симплектическую 2-форму $\Omega=d \theta$. Для каждого вектора $\xi$, принадлежащего алгебре Ли s группы Ли $S$, однопараметрическая подгруппа $\exp t \xi$ индуцирует гамильтоново векторное поле $\widehat{\xi}$ на $T^{*} N$ с гамильтонианом $\left.f_{\xi}=\theta(\widehat{\xi}): d f_{\xi}=-\widehat{\xi}\right\rfloor d \theta$. Следовательно, действие группы $S$ на $X=T^{*} N$ гамильтоново (пуассоново) [8], [18] и определяет отображение момента $\mathbf{J}: X \rightarrow \mathfrak{s}^{*}$ из $X$ в дуальное пространство к алгебре Ли $\mathfrak{s : ~} \mathbf{J}(x)(\xi)=f_{\xi}(x)$. Отображение $\mathbf{J}$ $S$-эквивариантно, т.е. оно сплетает действие $S$ на $X$ и коприсоединенное действие группы Ли $S$ на $\mathfrak{s}^{*}$.

Предположим, что группа $S$ компактна и действие $S$ на $N$ свободно и собственно. Тогда каждый элемент $\mu \in \mathfrak{s}^{*}$ является регулярным значением отображения $\mathbf{J}\left[18\right.$; предложение 2.2], в частности $\mathbf{J}^{-1}(\mu)$ - подмногообразие. Из соображений, упомянутых во введении, мы ограничим наше внимание подмногообразием $X_{0}=\mathbf{J}^{-1}(0)$. Многообразие $X_{0} G$-инвариантно. В самом деле, из определения 1-формы $\theta$ вытекает, что $x \in X_{0} \Leftrightarrow x\left(p_{*}\left(\widehat{\xi}_{x}\right)\right)=0 \forall \xi \in \mathfrak{s . ~ Н о ~ д е и ̆ с т в и я ~ г р у п п ~ Л и ~} G$ и $S$ на $N$ коммутируют, поэтому векторные поля $\widehat{\xi}, p_{*}(\widehat{\xi}) G$-инвариантны. Далее, вследствие эквивариантности многообразие $X_{0}$ инвариантно относительно действия группы $S$, а значит, пространство орбит $X_{0}^{\prime}=X_{0} / S$-корректно определенное многообразие и отображение $\pi: X_{0} \rightarrow X_{0}^{\prime}$ является главным $S$-расслоением. Так как его слои - это листы нулевого расслоения (ассоциированного с ограничением симплектической формы на $X_{0}$ ), то сушествует единственная симплектическая форма $\Omega_{0}^{\prime}$ на $X_{0}^{\prime}$ такая, что $\boldsymbol{\pi}^{*} \Omega_{0}^{\prime}=\mathbf{j}^{*} \Omega$, где $\mathbf{j}$ - отображение вложения $X_{0}$ в $X$. Так как действия групп Ли $G$ и $S$ на $X=T^{*} N$ коммутируют, то сушествует единственное действие группы $G$ на $X_{0}^{\prime}$ такое, что отображение $\pi G$-эквивариантно.

Пусть $\Omega^{\prime}=d \theta^{\prime}$ - каноническая симплектическая структура на $T^{*} N^{\prime}$, где $N^{\prime}=$ $N / S$.

ПРЕДЛОЖЕНИЕ 5.1 [18]. Редуцированное фазовое пространство $\left(X_{0}^{\prime}, \Omega_{0}^{\prime}\right)$ симплектоморфно многообразию $\left(T^{*} N^{\prime}, \Omega^{\prime}\right)$. Более того, при таком отожсдествлении $X_{0}^{\prime}$ с $T^{*} N^{\prime}$ мы имеем следующее тожсдество для канонических 1-форм: $\boldsymbol{\pi}^{*} \theta^{\prime}=\mathbf{j}^{*} \theta$.

Конструкция этого симплектоморфизма в [18] базируется на факте, что

$$
p_{S}^{*}\left(T^{*} N^{\prime}\right)=\mathbf{J}^{-1}(0)
$$

где $p_{S}: N \rightarrow N / S$ - каноническая субмерсия. Факторизуя по $S$ в $(5.1)$, получаем $T^{*} N^{\prime} \simeq X_{0}^{\prime}$. Так как действия $S$ и $G$ на $N$ коммутируют, то действие группы Ли $G$ на $X_{0}^{\prime} \simeq T^{*} N^{\prime}$ является продолжением естественного действия $G$ на факторпространстве $N / S$.

Пусть $F$ - положительно определенная $S$-инвариантная поляризация на $X$. 
ТЕОРемА 5.2 [8]. Существует канонически ассоциированная с $F$ положительно определенная поляризачия $F^{\prime}$ на редуцированном пространстве $X_{0}^{\prime}$.

Эта поляризация $F^{\prime}$ описывается следующим образом [8]. Для каждой точки $x \in X_{0}$ положим $F_{0}(x)=\left(T_{x}^{\mathbb{C}} X_{0}\right) \cap F(x)$. Тогда $F_{0}-S$-инвариантное подрасслоение (распределение) комплексифицированного касательного расслоения $T^{\mathbb{C}} X_{0}$ размерности $\left(\operatorname{dim} X_{0}^{\prime}\right) / 2$ и $F^{\prime}=\pi_{*}\left(F_{0}\right)$ (в частности, пересечение $F_{0}$ с комплексификацией ядра касательного отображения $\boldsymbol{\pi}_{*}$, порожденного векторными полями $\widehat{\xi} \mid X_{0}$, нулевое). Если распределение $F G$-инвариантно, то $F^{\prime}$ также $G$-инвариантно, потому что действия $G$ и $S$ на $N$ коммутируют.

5.2. Редуцированные кэлеровы структуры на $\mathbb{C} P^{n}$ и $\mathbb{H} P^{n}$. В этом пункте мы докажем, что все кэлеровы структуры $F(P), P=P(\psi, \varphi, \lambda)$ (для которых $\sigma$ является антиголоморфной инволюцией), на областях в касательных расслоениях симметрических пространств $\mathbb{C} P^{n}$ и $\mathbb{H} P^{n}$ могут быть получены с помощью процедуры редукции из аналогичных структур, определенных на областях касательных расслоений к сферам. Ниже мы рассмотрим распределения, отображения на этих трех типах многообразий и будем использовать обозначения, введенные ранее для $T(G / K)$, но с индексом $\mathbb{R}, \mathbb{C}, \mathbb{H}$ для сферы, комплексного и кватернионного проективных пространств соответственно.

Пусть $G^{\prime \prime}$ - группа Ли $\mathrm{SO}(2 n+2), n \geqslant 1$, и $K^{\prime \prime}$ - ее подгруппа, изоморфная $\mathrm{SO}(2 n+1)$. На $(2 n+1)$-мерной сфере $N=G^{\prime \prime} / K^{\prime \prime}$ транзитивно действует подгруппа Ли $G \subset G^{\prime \prime}$, изоморфная $\mathrm{SU}(n+1)$. Пересечение $G \cap K^{\prime \prime}-$ это подгруппа Ли $K \simeq \mathrm{SU}(n)$. Поэтому $N=G / K=\mathrm{SU}(n+1) / \mathrm{SU}(n)$. Комплексное проективное пространство $\mathbb{C} P^{n}$ - это однородное пространство $N^{\prime}=G / K^{\prime}=$ $\mathrm{SU}(n+1) / S(U(1) \times U(n))$, т.е. $N^{\prime}=N / S$, где $S$ - подгруппа Ли групшы $G$, изоморфиная $U(1)$ (мы рассматриваем действие групшы Ли $S$ справа). Рассмотрим также естественное действие группы $G$ на $N$ слева. Ясно, что эти два действия групп Ли $G$ и $S$ коммутируют. Пусть $\Phi_{\mathbb{R}}$ и $\Phi_{\mathbb{C}}-$ нормализованные билинейные формы $-\frac{1}{2} \operatorname{Tr}$ на вешественных полупростых алгебрах Ли $\mathfrak{g}^{\prime \prime}=\operatorname{so}(2 n+2)$ и $\mathfrak{g}=\operatorname{su}(n+1)$ соответственно (ассоциированные с точным стандартным представлением). Используя формы $\Phi_{\mathbb{R}}$ и $\Phi_{\mathbb{C}}$, мы отождествим кокасательные расслоения $T^{*} N=T^{*}\left(G^{\prime \prime} / K^{\prime \prime}\right)$ и $T^{*} N^{\prime}=T^{*}\left(G / K^{\prime}\right)$ с соответствующими касательньми расслоениями $X=T N$ и $X_{0}^{\prime}=T N^{\prime}$, как в п. 2.2. Пусть $\theta_{\mathbb{R}}$ и $\theta_{\mathbb{C}}^{\prime}$ обозначают канонические 1 -формы на $T N$ и $T N^{\prime}$ (зависящие от этих отождествлений). Обозначим через $H_{\mathbb{R}}$ и $H_{\mathbb{C}}^{\prime}$ соответствующие формам $\Phi_{\mathbb{R}}$ и $\Phi_{\mathbb{C}}$ гамильтонианы геодезических потоков на многообразиях $T N=T\left(G^{\prime \prime} / K^{\prime \prime}\right)$ и $T N^{\prime}=T\left(G / K^{\prime}\right)$. Но, отождествляя $T^{*} N=T^{*}(G / K)$ с $T N=T(G / K)$ с помошью формы $\Phi_{\mathbb{C}}$, мы получаем другие каноническую 1-форму $\theta_{\mathbb{C}}$ и гамильтониан $H_{\mathbb{C}}$ на $T N$. Ясно, что $C_{\mathbb{R} \mathbb{C}} \theta_{\mathbb{C}}=\theta_{\mathbb{R}}$ и $C_{\mathbb{R} \mathbb{C}} H_{\mathbb{C}}=H_{\mathbb{R}}$, где $C_{\mathbb{R} \mathbb{C}}-$ некоторая константа.

Пусть $\mathfrak{g}=\mathfrak{k} \oplus \mathfrak{s} \oplus \mathfrak{m}^{\prime}$ - разложение алгебры Ли $\mathfrak{g}$ в прямую сумму ортогональных относительно формы $\Phi_{\mathbb{C}}$ подпространств, где $\mathfrak{k}$ - алгебра Ли подгруппы Ли $K \subset G$. Пусть $\mathfrak{m}=\mathfrak{s} \oplus \mathfrak{m}^{\prime}$. Обозначим через $\mathfrak{k}^{\prime}$ алгебру Ли группы Ли $K^{\prime} \subset G: \mathfrak{k}^{\prime}=\mathfrak{k} \oplus \mathfrak{s}$. Так как $\left[\mathfrak{k}^{\prime}, \mathfrak{m}\right] \subset \mathfrak{m}$, то мы можем рассмотреть тривиальное векторное расслоение $G \times \mathfrak{m}$ с действием на нем групгы Ли $K^{\prime}$ справа $r_{k^{\prime}}:(g, w) \mapsto\left(g k^{\prime}, \operatorname{Ad} k^{\prime-1}(w)\right)$. Пусть $\mathbf{J}: T(G / K) \rightarrow \mathfrak{s}^{*}$ - отображение момента, ассоциированное с (правым) действием группы Ли $S$ на $N=G / K$. Используя (лево) $G$-эквивариантную субмер- 
сию $\Pi: G \times \mathfrak{m} \rightarrow T(G / K),\left.(g, w) \mapsto \frac{d}{d t}\right|_{0} g \exp (t w) K$, и лемму 2.3, мы получаем, что $(\mathbf{J} \circ \Pi)(g, w)(\eta)=\widetilde{\theta}_{\mathbb{C}(g, w)}\left(\eta^{l}(g),[w, \eta]\right)=\Phi_{\mathbb{C}}(w, \eta)$ для произвольных $\eta \in \mathfrak{s}$. Поэтому П $\left(G \times \mathfrak{m}^{\prime}\right)=\mathbf{J}^{-1}(0)=X_{0}$, а значит, $X_{0}^{\prime}=T N^{\prime} \simeq G \times_{K^{\prime}} \mathfrak{m}^{\prime}$. Более того, из $(2.2)$ вытекает, что $\boldsymbol{\pi}^{*} \theta_{\mathbb{C}}^{\prime}=\mathbf{j}^{*} \theta_{\mathbb{C}}$. Так как отображение $T(G / K) \rightarrow T\left(G^{\prime \prime} / K^{\prime \prime}\right)$, $\left.g \exp (t \xi) K \mapsto \frac{d}{d t}\right|_{0} g \exp (t \xi) K^{\prime \prime}, g \in G, \xi \in \mathfrak{m}$, является диффеоморфизмом $(G$ действует транзитивно на $\left.G^{\prime \prime} / K^{\prime \prime}\right)$ и $H_{\mathbb{R}}=C_{\mathbb{R} \mathbb{C}} H_{\mathbb{C}}$, редуцированный гамильтониан $H_{\mathbb{R}}^{\prime}$ на $X_{0}^{\prime}$ совпадает с $C_{\mathbb{R} \mathbb{C}} H_{\mathbb{C}}^{\prime}$. Чтобы найти константу $C_{\mathbb{R} \mathbb{C}}$, рассмотрим стандартное вложение $\mathrm{su}(n+1) \rightarrow \mathrm{so}(2 n+2), \xi=(A+i B) \mapsto \xi^{\prime \prime}=\left(\begin{array}{cc}A & -B \\ B & A\end{array}\right)$, алгебры Ли $\mathfrak{g}$ в алгебру Ли $\mathfrak{g}^{\prime \prime}$. Пусть $\mathfrak{k}^{\prime \prime}=\operatorname{so}(2 n+1)$ - алгебра Ли подгрупшы $K^{\prime \prime} \subset G^{\prime \prime}$. Так как для билинейных (след)-форм (на $\mathfrak{g}$ и $\left.\mathfrak{g}^{\prime \prime}\right) \operatorname{Tr} \xi^{2}=\operatorname{Tr}\left(\xi^{\prime \prime}\right)_{\mathfrak{m}^{\prime \prime}}^{2}$, где $\xi \in \mathfrak{m} \subset \mathfrak{g}$ и $(\cdot)_{\mathfrak{m}^{\prime \prime}}-$ проекция на $\mathfrak{m}^{\prime \prime}$ вдоль $\mathfrak{k}^{\prime \prime}$ в $\mathfrak{g}^{\prime \prime}$, определенная прямым ортогональным разложением $\mathfrak{g}^{\prime \prime}=\mathfrak{k}^{\prime \prime} \oplus \mathfrak{m}^{\prime \prime}[14]$, мы получим, что $C_{\mathbb{R} \mathbb{C}}=1$. Отметим, что $\operatorname{Tr} \xi^{2}=\frac{1}{2} \operatorname{Tr}\left(\xi^{\prime \prime}\right)^{2}$.

Пусть $F_{\mathbb{R}}^{c}-G^{\prime \prime}$-инвариантная каноническая кэлерова структура на $X=$ $T\left(G^{\prime \prime} / K^{\prime \prime}\right)$ со свойствами $(1),(2)$ теоремы 4.15 (S-инвариантная в силу этой же теоремы). Такая глобальная структура имеет вид $F_{\mathbb{R}}(P)$, где $P=P(\psi, \psi, 1)$ и $\psi(\mathbf{r})=\mathbf{r} \operatorname{cth}(\mathbf{r})$ (см. доказательство теоремы 4.14$)$. В силу теоремы 5.2 сушествует редуцированная кэлерова структура $F_{\mathbb{R}}^{\prime}$ на $X_{0}^{\prime}=T N^{\prime}$ такая, что $F_{\mathbb{R}}^{\prime}=\pi_{*}\left(F_{\mathbb{R} 0}^{c}\right)$, т.е. $\boldsymbol{\pi}^{*}\left(\operatorname{Im} \bar{\partial} H_{\mathbb{R}}^{\prime}\right)=\mathbf{j}^{*}\left(\operatorname{Im} \bar{\partial} H_{\mathbb{R}}\right)$ (чтобы доказать это, достаточно использовать определение $\bar{\partial}: \quad \bar{\partial} H|F=d H| F, \bar{\partial} H \mid \bar{F}=0)$. Но $\boldsymbol{\pi}^{*} \theta_{\mathbb{C}}^{\prime}=\mathbf{j}^{*} \theta_{\mathbb{C}}$, поэтому $\operatorname{Im} \bar{\partial} H_{\mathbb{R}}^{\prime}=C_{\mathbb{R} \mathbb{C}} \theta_{\mathbb{C}}^{\prime}$ - каноническая 1-форома на $T N^{\prime}$ с точностью до константы $C_{\mathbb{R C}}=1$. Применяя теорему 4.15 и аргументы доказательства теоремы 4.14 , мы получаем, что кэлерова структура $F_{\mathbb{R}}^{\prime}$ совпадает с канонической (глобальной) структурой $F_{\mathbb{C}}^{c}$ на редуцированном пространстве $X_{0}^{\prime}=T\left(G / K^{\prime}\right)$.

Пусть $F_{\mathbb{R}}=F_{\mathbb{R}}(P)$ - произвольная $G^{\prime \prime}$-инвариантная кэлерова структура на некоторой $\sigma-, G^{\prime \prime}$ - и $S$-инвариантной области $D_{\mathbb{R}} \subset T\left(G^{\prime \prime} / K^{\prime \prime}\right)$. Предположим, что $\sigma: X \rightarrow X$ - антиголоморфная инволюция, т.е. $P=P(\psi, \psi, \lambda)$, где $\psi, \lambda$ - положительные функции. Существует (локальная) положительная функция $\delta=\delta(\mathbf{r})$, для которой $\lambda(\mathbf{r})=1 /(\mathbf{r} \delta(\mathbf{r}))^{\prime}$. В силу (3.4), (4.5) (см. также (3.9)) при подходящем выборе постоянной интегрирования мы (локально) получим $\Psi_{\mathbb{R} *}(\gamma)\left(F_{\mathbb{R}}^{c}\right)=F_{\mathbb{R}}(P)$ (в частности, $F_{\mathbb{R}}(P) S$-инвариантна). По определению (локальный) диффеоморфизм $\Psi_{\mathbb{R}}(\gamma)$ отображает точку $\left(g^{\prime \prime}, Y\right) \in T\left(G^{\prime \prime} / K^{\prime \prime}\right)$ в $\left(g^{\prime \prime}, \gamma\left(\sqrt{H_{\mathbb{R}}(Y)}\right) Y\right)$. Значит, этот диффеоморфизм оставляет инвариантным подмногообразие $X_{0}$. Более того, так как $H_{\mathbb{R}}=H_{\mathbb{C}}$, то мы имеем $\boldsymbol{\pi} \circ \Psi_{\mathbb{R}}(\gamma)=\Psi_{\mathbb{C}}^{\prime}(\gamma) \circ \boldsymbol{\pi}$, где (локальный) диффеоморфизм $\Psi_{\mathbb{C}}^{\prime}(\gamma)$ на $X_{0}^{\prime}=T\left(G / K^{\prime}\right)$ определяется с помощью $H_{\mathbb{C}}^{\prime}$. В силу теоремы 5.2 существует редуцированная кэлерова структура $F_{\mathbb{R}}^{\prime}$ на некотором открытом подмножестве в $X_{0}^{\prime}=T N^{\prime}$ такая, что $F_{\mathbb{R}}^{\prime}=\pi_{*}\left(F_{\mathbb{R} 0}\right)$. Таким образом, $F_{\mathbb{R}}^{\prime}=\Psi_{\mathbb{C} *}^{\prime}(\gamma)\left(F_{\mathbb{C}}^{c}\right)$ (локально), т.е. эта структура имеет вид $F_{\mathbb{C}}(P)$, где $P=P(\psi, \varphi, \lambda)$. Доказана

Tеорема 5.3. Пусть $N=S^{2 n+1}$ u $F_{\mathbb{R}}(P), P=P(\psi, \psi, \lambda)$, - кәлерова структура на $D_{\mathbb{R}} \subset\left(T N, d \theta_{\mathbb{R}}\right), D_{\mathbb{R}}=\left\{x \in T N: H_{\mathbb{R}}(x) \in O\right\}$, для которой $\sigma$ является антиголоморфной инволюиией. Редуцированное многообразие $\mathbf{J}^{-1}(0) / S$, әде $S \simeq U(1)$, изоморфно $\left(T N^{\prime}, d \theta_{\mathbb{C}}^{\prime}\right), N^{\prime}=\mathbb{C} P^{n}$, с редуиированной кәлеровой структурой $F_{\mathbb{R}}^{\prime}=F_{\mathbb{C}}(P)$ на $D_{\mathbb{C}}^{\prime}=\left\{x^{\prime} \in T N^{\prime}: H_{\mathbb{C}}^{\prime}\left(x^{\prime}\right) \in O\right\}$, где $P=P(\psi, \varphi, \lambda)$ $u \varphi(\mathbf{r})=\psi(\mathbf{r})+\mathbf{r}^{2} / \psi(\mathbf{r})$, если $n \geqslant 2, P=P(\psi, \psi, \lambda)$, если $n=1$. 
Теперь пусть $G^{\prime \prime}$ - группа Ли $\mathrm{SO}(4 n+4), n \geqslant 1$, и $K^{\prime \prime}$ - ее подгруппа, изоморфная $\mathrm{SO}(4 n+3)$. На $(4 n+3)$-мерной сфрере $N=G^{\prime \prime} / K^{\prime \prime}$ транзитивно действует подгруппа $G \subset G^{\prime \prime}$, изоморфная $\operatorname{Sp}(n+1)$. Пересечение $G \cap K^{\prime \prime}$ - это подгруппа Ли $K \simeq \operatorname{Sp}(n)$. Поэтому $N=\operatorname{Sp}(n+1) / \operatorname{Sp}(n)$. Кватернионное проективное пространство $\mathbb{H} P^{n}$ определяется как однородное пространство $N^{\prime}=\operatorname{Sp}(n+1) /(\operatorname{Sp}(1) \times \operatorname{Sp}(n))$, т.е. $N^{\prime}=N / S$, где $S-$ подгруппа Ли групшы $G$, изоморфная $\mathrm{Sp}(1)$ (мы рассматриваем действие $S$ справа). Пусть $\Phi_{\mathbb{R}}$ и $\Phi_{\mathbb{H}}$ обозначают нормализованные билинейные формы $-\frac{1}{2} \operatorname{Tr}$ на полупростых алгебрах Ли $\mathfrak{g}^{\prime \prime}=\mathrm{so}(4 n+4)$ и $\mathfrak{g}=\mathrm{sp}(n+1)$ соответственно (ассоциированные с точными стандартньми представлениями вещественными и кватернионными матрицами соответственно). Используя формы $\Phi_{\mathbb{R}}$ и $\Phi_{\mathbb{H}}$, мы отождествим кокасательные расслоения $T^{*} N=T^{*}\left(G^{\prime \prime} / K^{\prime \prime}\right)$ и $T^{*} N^{\prime}=T^{*}\left(G / K^{\prime}\right)$ с соответствующими касательньми расслоениями $X=T N$ и $X_{0}^{\prime}=T N^{\prime}$, как в п. 2.2. Пусть $\theta_{\mathbb{R}}$ и $\theta_{\mathbb{H}}^{\prime}$ - канонические 1-формы на $T N$ и $T N^{\prime}$ (зависящие от этих отождествлений). Мы находимся в ситуации, аналогичной рассмотренной выше в случае пары $\left(S^{2 n+1}, \mathbb{C} P^{n}\right)$. Заменяя, где это необходимо, $\mathbb{C} \mapsto \mathbb{H}$ и вычисляя $C_{\mathbb{R} H}=1$, завершаем доказательство следуюшей теоремы.

TеOPEMA 5.4. Пусть $N=S^{4 n+3}$ u $F_{\mathbb{R}}(P), P=P(\psi, \psi, \lambda)$, - кәлерова структура на $D_{\mathbb{R}} \subset\left(T N, d \theta_{\mathbb{R}}\right), D_{\mathbb{R}}=\left\{x \in T N: H_{\mathbb{R}}(x) \in O\right\}$, для которой $\sigma$ является антиголоморфной инволюиией. Редуцированное многообразие $\mathbf{J}^{-1}(0) / S$, где $S \simeq \operatorname{Sp}(1)$, изоморфно $\left(T N^{\prime}, d \theta_{\mathbb{H}}^{\prime}\right)$, әде $N^{\prime}=\mathbb{H} P^{n}$, с редуиированной кәлеровой структурой $F_{\mathbb{R}}^{\prime}=F_{\mathbb{H}}(P)$ на $D_{\mathbb{H}}^{\prime}=\left\{x^{\prime} \in T N^{\prime}: H_{\mathbb{H}}^{\prime}\left(x^{\prime}\right) \in O\right\}$, где $P=P(\psi, \varphi, \lambda)$ $u \varphi(\mathbf{r})=\psi(\mathbf{r})+\mathbf{r}^{2} / \psi(\mathbf{r})$, если $n \geqslant 2, P=P(\psi, \psi, \lambda)$, если $n=1$.

ЗАмечАниЕ 5.5. Полезно отметить, что вся конструкция целиком и доказательства теорем вьше не используют компактность пространства $G^{\prime \prime} / K^{\prime \prime}$. Поэтому аналогичные теоремы могут быть доказаны и в некомпактном случае с использованием этой же конструкции.

\section{Список литературы}

1. Souriau J. M. Sur la variété de Kepler // Sympos. Math. 1974. V. 14. P. 343-360.

2. Rawnsley J. H. Coherent states and Kähler manifolds // Quart. J. Math. Oxford Ser. (2). 1977. V. 28. P. 403-415.

3. Furutani K., Tanaka R. A Kähler structure on the punctured cotangent bundle of complex and quaternion projective spaces and its applications to geometric quantization. I // J. Math. Kyoto Univ. 1994. V. 34. № 4. P. 719-737.

4. Ii K., Morikawa T. Kähler structures on the tangent bundle of Riemannian manifolds of constant positive curvature // Bull. Yamagata Univ. Natur. Sci. 1999. V. 14. №3. P. 141-154.

5. Szőke R. Adapted complex structures and geometric quantization // Nagoya Math. J. 1999. V. 154. P. 171-183.

6. Mykytyuk I. V. Invariant Kähler structures on the cotangent bundle of compact symmetric spaces // Submitted to Nagoya Math. J.

7. Gawedzki K. Fourier-like kernels in geometric quantization // Dissertationes Math. (Rozprawy Mat.). 1976. V. 128. P. 1-80.

8. Guillemin V., Sternberg S. Geometric quantization and multiplicities of group representations // Invent. Math. 1982. V. 67. № 3. P. 515-538.

9. Guillemin V., Stenzel $M$. Grauert tubes and the homogeneous Monge-Ampere equation. II // J. Differential Geom. 1992. V. 35. №3. P. 627-641. 
10. Mostow G. D. Some new decomposition theorems for semisimple groups // Mem. Amer. Math. Soc. 1955. V. 14. P. 31-54.

11. Guillemin V., Stenzel $M$. Grauert tubes and the homogeneous Monge-Ampere equation // J. Differential Geom. 1991. V. 34. № 2. P. 561-570.

12. Dancer A., Szőke R. Symmetric spaces, adapted complex structures and hyperkähler structures // Quart. J. Math. Oxford Ser. (2). 1997. V. 48. № 2. P. 27-38.

13. Szőke $R$. Adapted complex structures and Riemannian homogeneous spaces // Ann. Polon. Math. 1998. V. 70. P. 215-220.

14. Helgason S. Differential geometry, Lie groups, and symmetric spaces. New York: Academic Press, 1978. (Pure Appl. Math. V. 80.)

15. Гото М., Гроссханс Ф. Полупростые алгебры Ли. М.: Мир, 1981.

16. Onishchik A. L. Topology of transitive transformation groups. Leipzig: Johann Ambrosius Barth, 1994.

17. Постников М. М. Лекции по геометрии. Группы и алгебры Ли. М.: Наука, 1982.

18. Gotay M. Constraints, reduction and quantization // J. Math. Phys. 1986. V. 27. № 8 . P. 2051-2066.

Государственный университет "Львовская политехника",

Львов, Украина

Поступила в редакцию

E-mail: viva@iapmm.lviv.ua 